\title{
Bioconversion of Lignocellulosic Biomass into Value Added Products under Anaerobic Conditions: Insight into Proteomic Studies
}

\author{
Martha Inés Vélez-Mercado ${ }^{1}{ }^{\mathbb{D}}$, Alicia Guadalupe Talavera-Caro ${ }^{1}$, Karla María Escobedo-Uribe ${ }^{1}$, \\ Salvador Sánchez-Muñoz ${ }^{2}$, Miriam Paulina Luévanos-Escareño ${ }^{1}$ (D), Fernando Hernández-Terán ${ }^{1}$, \\ Alejandra Alvarado ${ }^{3, *(D)}$ and Nagamani Balagurusamy ${ }^{1, *(D)}$
}

1 Laboratorio de Biorremediación, Facultad de Ciencias Biológicas, Ciudad Universitaria de la Universidad Autónoma de Coahuila, Carretera Torreón-Matamoros km. 7.5, Torreón CP. 27276, Mexico; marthavelez@uadec.edu.mx (M.I.V.-M.); alicia_talavera@uadec.edu.mx (A.G.T.-C.); karla.uribe@uadec.edu.mx (K.M.E.-U.); miriam_luevanos@uadec.edu.mx (M.P.L.-E.); fernandohernandezteran@uadec.edu.mx (F.H.-T.)

2 Bioprocesses and Sustainable Products Laboratory, Department of Biotechnology, Engineering School of Lorena, University of São Paulo (EEL-USP), Lorena 12602-810, SP, Brazil; salvador.sanchez@usp.br

check for

updates

Citation: Vélez-Mercado, M.I.;

Talavera-Caro, A.G.;

Escobedo-Uribe, K.M.;

Sánchez-Muñoz, S.;

Luévanos-Escareño, M.P.;

Hernández-Terán, F.; Alvarado, A.;

Balagurusamy, N. Bioconversion of

Lignocellulosic Biomass into Value

Added Products under Anaerobic

Conditions: Insight into Proteomic Studies. Int. J. Mol. Sci. 2021, 22, 12249. https://doi.org/10.3390/ ijms222212249

Academic Editors: Diby Pau and Udai B. Singh

Received: 31 August 2021

Accepted: 9 November 2021

Published: 12 November 2021

Publisher's Note: MDPI stays neutral with regard to jurisdictional claims in published maps and institutional affiliations.

Copyright: (C) 2021 by the authors. Licensee MDPI, Basel, Switzerland This article is an open access article distributed under the terms and conditions of the Creative Commons Attribution (CC BY) license (https:// creativecommons.org/licenses/by/ $4.0 /)$
3 Interfaculty Institute for Microbiology and Infection Medicine Tübingen, University of Tübingen, Auf der Morgenstelle 24, 72076 Tübingen, Germany

* Correspondence: a.alvarado@cmfi.uni-tuebingen.de (A.A.); bnagamani@uadec.edu.mx (N.B.)

Abstract: Production of biofuels and other value-added products from lignocellulose breakdown requires the coordinated metabolic activity of varied microorganisms. The increasing global demand for biofuels encourages the development and optimization of production strategies. Optimization in turn requires a thorough understanding of the microbial mechanisms and metabolic pathways behind the formation of each product of interest. Hydrolysis of lignocellulosic biomass is a bottleneck in its industrial use and often affects yield efficiency. The accessibility of the biomass to the microorganisms is the key to the release of sugars that are then taken up as substrates and subsequently transformed into the desired products. While the effects of different metabolic intermediates in the overall production of biofuel and other relevant products have been studied, the role of proteins and their activity under anaerobic conditions has not been widely explored. Shifts in enzyme production may inform the state of the microorganisms involved; thus, acquiring insights into the protein production and enzyme activity could be an effective resource to optimize production strategies. The application of proteomic analysis is currently a promising strategy in this area. This review deals on the aspects of enzymes and proteomics of bioprocesses of biofuels production using lignocellulosic biomass as substrate.

Keywords: lignocellulose substrates; biofuels; value added products; anaerobic conditions; proteomics

\section{Introduction}

Biofuels are gaining attention due to the environmental concerns caused by the increasing emissions of greenhouse gases. Although biofuels are practical alternatives to replace fossil fuels, edible sources (soybean, rapeseed, etc.) are frequently used as substrates, which could limit the possibility of meeting the growing energy demand [1]. Hence, alternative biofuels from non-edible substrates have garnered increased attention [2] Plant-derived biomass (i.e., lignocellulose) is the most abundant sustainable source and promising feedstock to produce biofuels (e.g., bioethanol, biobutanol, and biodiesel) and other value-added products (e.g., biomaterials and biochemicals) [3,4].

In general, aerobic bioconversion of lignocellulolytic substrates and their microbiology have been extensively studied. Mostly, fungi are employed due to their extracellular cellulases, xylanases and ligninase enzymes and their activity potential [5]. Additionally, 
their mechanisms of degradation involve less steps. Since lignin degradation is mediated by the incorporation of oxygen atom to facilitate cleavage of the aromatic ring, various strategies such as bioreactors design, increased oxygen transfer mechanisms, etc. have been implemented to improve the efficiency of the bioconversion process [6,7].

However, anaerobic breakdown of lignocellulose requires a consortium of several microorganisms, where lignin degradation and cellulose hydrolysis are important ratelimiting steps. Anaerobic cellulolytic microorganisms employ cellulosome, a protein complex that allows binding to the raw substrates and enhances hydrolysis of polysaccharides (more in Section 3.1.). Cellulosome activity is well studied in many Clostridium bacteria. A notable example is the strain $C$. thermocellum, which has been deemed a promising candidate for biotechnological applications [8]. Earlier, Shinoda et al. [9] compared two different strains of cellulosome-producing clostridia, viz., C. thermocellum and C. clariflavum, and concluded that $C$. thermocellum showed a cellulolytic activity of $4.1 \mathrm{U} / \mathrm{mg}$ with phosphoric acid swollen cellulose and $0.35 \mathrm{U} / \mathrm{mg}$ with avicel, while $C$. clariflavum recorded 2.6 and $0.16 \mathrm{U} / \mathrm{mg}$, respectively. Conversely, they reported that $C$. clariflavum demonstrated higher hemicellulolytic activity of $2.4 \mathrm{U} / \mathrm{mg}$ with xyloglucan and $1.7 \mathrm{U} / \mathrm{mg}$ with mannan, and $C$. thermocellum registered 1.6 and $1.3 \mathrm{U} / \mathrm{mg}$, respectively. Moreover, recent work where $C$. thermocellum expressed $\beta$-glucosidase from a heterologous system, indicated that collective activity of cellulosome enzymes and $\beta$-glucosidases positively increases cellulose hydrolysis by this bacterium [10]. Additionally, C. thermocellum have the metabolic pathway to produce ethanol as a one-step process directly from cellulose, and a maximum theoretical ethanol yield of $75 \%$ can be obtained [7]. C. thermocellum have been reported in anaerobic digesters as well and have been related with increases in methane production [11].

As mentioned previously, one of the major bottlenecks is due to the complex structure and recalcitrance of lignin [12], and many authors suggest that pretreatment could aid in removal of $20-60 \%$ lignin fraction, which is dependent on solids content, enzyme activity, etc. A yield of about $60-80 \%$ sugar has been reported after the pretreatment steps [13-15]. The efficiency and yield in the bioconversion of lignocellulosic biomass into biofuels and other value-added processes under anaerobic conditions depend on the development of yield efficient and cost-effective lignin removal processes.

Multi-omics analyses, viz., combination of metagenomic, proteomics, transcriptomics, metabolomics offer important tools in deciphering the microbial diversity, identification of key proteins and designing of suitable microbial consortia for production of biofuels and other value added bioproducts. In particular, proteome provides the measurement of expression and activity state of proteins in a cell $[16,17]$. Through proteomics, data on the structural and functional elements present in the cell and as well as their molecular interactions in biological processes are obtained [18]. In the context of biofuel production, proteomics has been integrated to complement the understanding and regulation of cellular processes, to identify biomarkers for monitoring and to evaluate scaling-up options [19-21]. Further, proteomics studies are mainly focused on identifying proteins associated with plant polysaccharide depolymerization [22,23], stress tolerance and metabolic responses to varied treatments [24-26]. Additionally, genomic technologies facilitate the design and modification of microbial strains to obtain increased efficiency and yield [27-29].

This review focuses on role of different proteins and proteomic insights on the anaerobic bioconversion of lignocellulose substrates for biofuel production and other value-added products.

\section{Importance of Proteomic Technologies in Bioprocesses}

The use of lignocellulosic feedstocks for production of biofuels and other chemicals has gained strength over time. The characterization of lignocellulose and a detailed understanding of its degradation process is critical. Some of the key proteins that have been identified in lignocellulose degradation are cellulases [30], xylanases [31], peroxidases and laccases [32], as well as glycoside hydrolases or GHs [33,34]. Transcriptomics, proteomics, 
chemoproteomics and metabolomics are used to map, measure or sequence biomolecules from microbial communities. These technologies aid in gathering information related to novel genes, gene functionality, genomic structure, metabolic pathways, and the evolutionary history of the microorganisms implicated in biofuel production. In brief, genomic and transcriptomic technologies help in understanding the genetic elements and their regulation (DNA \& RNA), while proteomics provides information on the structural and functional characterization of protein products $[17,35]$. The obtained molecular information contributes to the development of novel strategies to recover resources and energy from recalcitrant substrates to meet the biofuel demands of the future generations [3].

Some proteomic samples tend to be complex and have an abundance of different components, which makes gel-based techniques (2D PAGE) unsuitable for their analysis. However, there are other methods that can be used for high-throughput proteomics, such as LC-MS/ MS, which generally has two different approaches differentiated by upstream sample preparation methods. The most common approach is bottom-up proteomics, where the protein samples are digested prior to the LC-MS/ MS analysis (1D, 2D and Multidimensional LC can be used). Top-down proteomics is another suitable alternative in which proteins are not digested and are directly analyzed by LC-MS / MS [36] (Figure 1A). In addition, isobaric tags for relative, and absolute quantitation (iTRAQ) can be used for comparative proteomics to identify the different relative intensity proteins associated with stress conditions and/ or increased biofuel yield [37]. Together, these approaches facilitate the identification of key proteins and their production levels during lignocellulose degradation and fermentation (Figure 1B).

A)

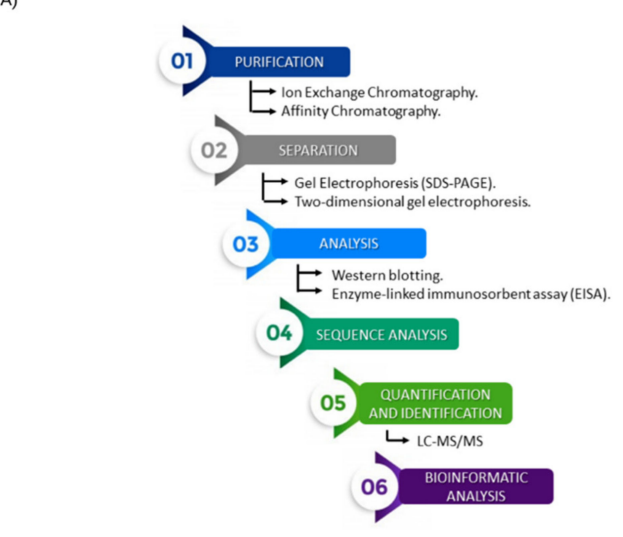

B)

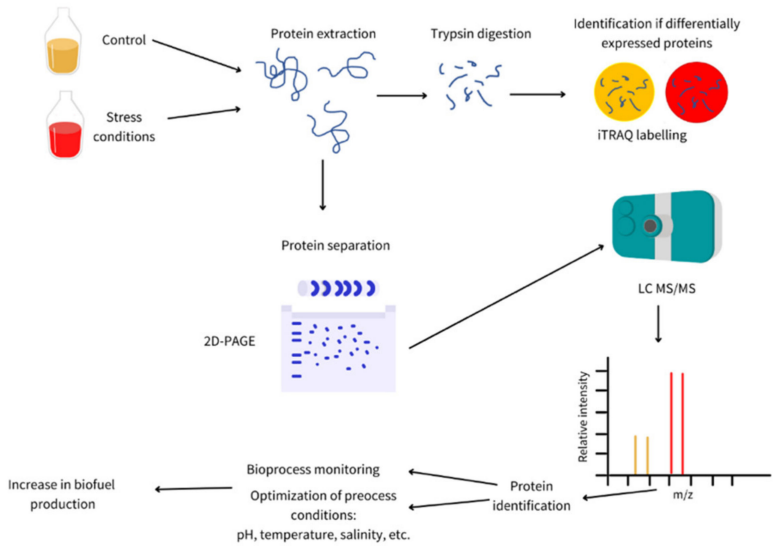

Figure 1. (A) Schematic outline for the identification of proteins. (B) Optimization and monitoring of bioprocess through the identification of functional proteins.

\section{Proteins Involved in Lignocellulose Utilization}

Lignocellulosic biomass is mainly composed of $40-60 \%$ cellulose, $20-40 \%$ hemicellulose, and 10-24\% lignin, but in general, composition varies in different cell walls depending 
on the plant species [38]. Different degradative enzymes are implicated in the breakdown of the lignocellulosic structure and the hydrolysis or oxidation of the polymers present in plant-derived biomass [39].

The recalcitrant nature of lignocellulose [40] makes its hydrolysis a limiting step, and thus converting lignocellulose into biofuel could be an expensive process [41]. Thus, facilitating hydrolysis and subsequent polysaccharide conversion is critical to improve biofuels production feasibility and competitivity against other fuel alternatives. Most of the proteomics studies on the degradation of lignocellulosic biomass to obtain value-added products has been primarily employed to determine hydrolytic enzymes, particularly, the extracellular enzymes secreted by microorganisms (Figure 2C), which together are known as the secretome [42]. The simple sugars obtained after the hydrolysis of polysaccharides by secretome enzymes are readily utilized as a carbon source for biofuel [43]. Different enzymes that participate in hydrolysis of lignocellulosic substrates and their mechanisms are presented in this section.
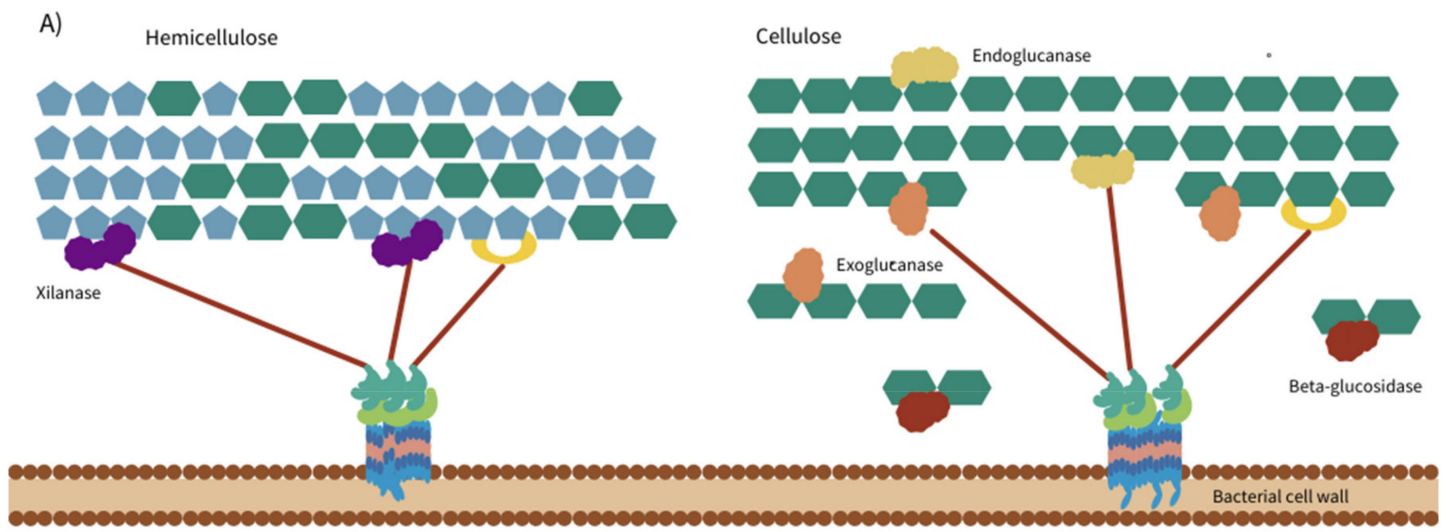

B)
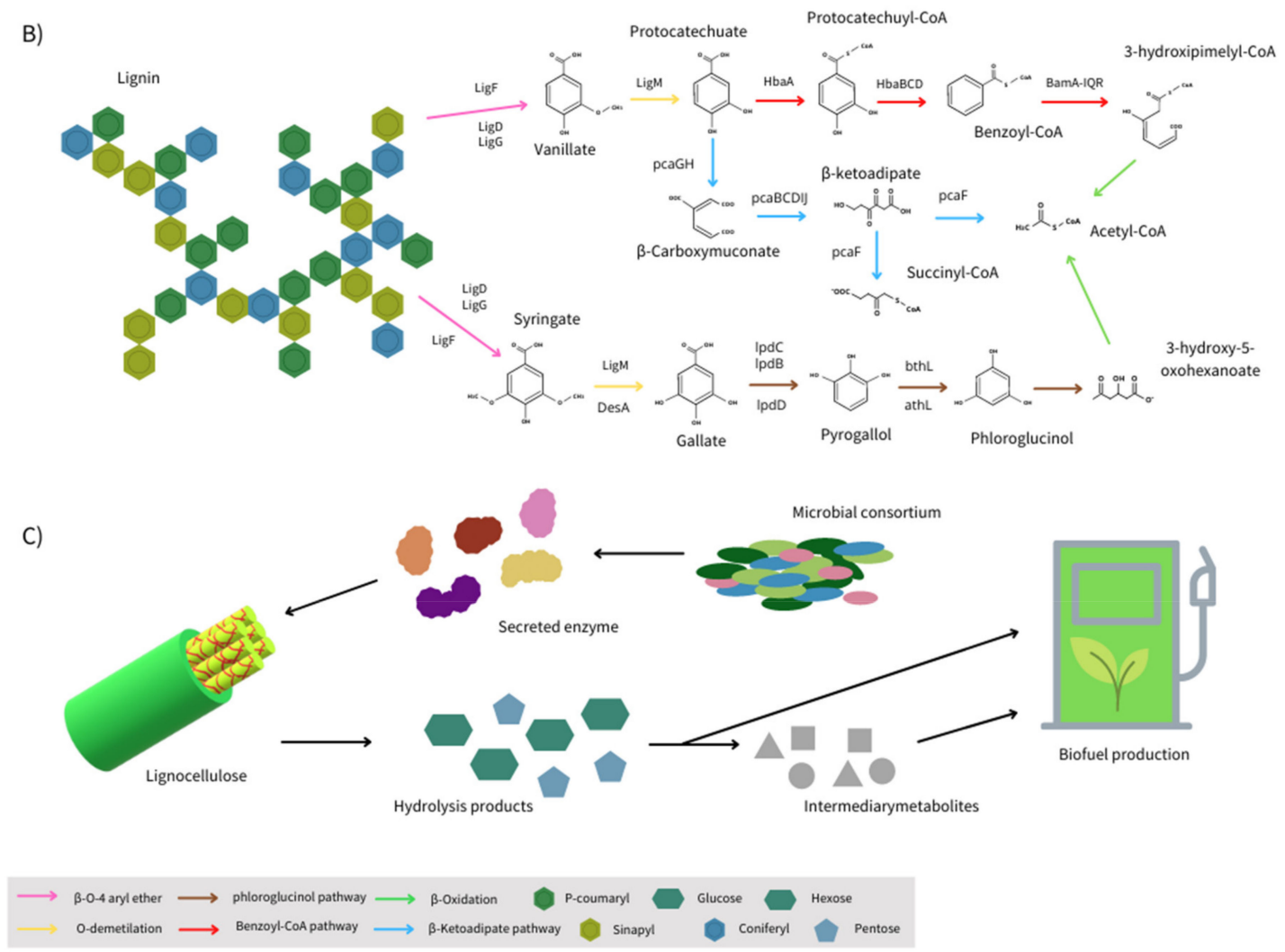

Figure 2. (A) Enzymatic mechanisms of the degradation of polysaccharides, (B) lignin degradation and (C) its potential for the production of by-products. 


\subsection{Enzymes Targeting Lignocellulosic Polysaccharides}

Cellulose, one of the major components of lignocellulose, is a homopolysaccharide with amorphous and crystalline regions [44] made of glucose monomers linked by $\beta$-1,4-Dglucan. Hemicellulose however is a heterogenous polysaccharide conformed by a xylan backbone that contains xylose, arabinose, mannose, glucose, galactose and sugar acids in different proportions depending on the source $[43,45]$.

Hydrolytic enzymes capable of acting on cellulose and hemicellulose are called cellulolytic and hemicellulolytic enzymes, respectively, and belong to glycoside hydrolases $(\mathrm{GH})$, which are grouped under CAZy (Carbohydrate-Active enZymes). GHs are classified according to its primary sequence into 168 families in the CAZy database [46]. A given GH enzyme may belong to a particular family based on its specific characteristics, such as protein structure, enzymatic activity, specificity, and reaction mechanism [47]. In general, two different mechanisms, viz., inversion and retention, are employed by GH families to cleave glycosidic bonds [48,49].

Three cellulolytic enzymes (exoglucanase, endoglucanase, $\beta$-glucosidase) play a major role in cellulose biodegradation. Exoglucanases (EC 3.2.1.91) as well as endoglucanase (EC 3.2.1.4) employ either inverting or retaining mechanisms to hydrolyze the $\beta-1,4$ linkages of cellulose in amorphous and crystalline regions, respectively. Meanwhile, $\beta$-glucosidase (EC 3.2.1.21) act synergistically with exoglucanases and endoglucanases, by hydrolyzing the $\beta-1,4$ linkages of a cellulose-derived disaccharide (i.e., cellobiose) [50,51]. The major enzymes involved in cellulose hydrolysis expressed by a variety of microorganisms; especially, Clostridium genera are described in Table 1.

A greater number of enzymes are involved in the degradation of hemicellulose due to its heterogenous structure. In addition to $\mathrm{GH}$, hemicellulases include carbohydrate esterases (CEs). Since xylan is the major component of hemicellulose, xylanases (EC 3.2.1.8) are one of the main enzymes involved in hemicellulose depolymerization by cleavage of the $\beta-1,4$ linkages of the xylan backbone, producing xylooligomers such as xylobiose and xylose. Besides xylanases, $\beta$-Xylosidases, $\alpha$-L-arabinofuranosidases, $\beta$-mannanases, $\beta$-mannosidases and $\alpha$-glucuronidases also play an important role in the breakdown of hemicellulose and have been identified by proteomic analyses by several authors (Tables 1 and 2). It can be seen from Tables 1 and 2 that there are multiple reports on anaerobic hydrolysis of cellulosic biomass. However, in the case of lignin degradation, the available reports are on aerobic bioconversion of lignin.

Table 1. Enzymes involved in depolymerization of different polysaccharide substrates and their microbial source.

\begin{tabular}{|c|c|c|c|c|c|c|}
\hline & EC Number & Putative Function & Organism Source & Substrate & Activity or Function & References \\
\hline Cellulose & 3.2.1.21 & $\beta$-glucosidase & $\begin{array}{c}\text { Bacteroides coprosuis } \\
\text { Roseburia intestinails } \\
- \\
\text { Clostridium termitidi } \\
- \\
\text { Pantoea ananatis Sd-1 }\end{array}$ & $\begin{array}{c}\text { Corn stover } \\
- \\
\alpha \text {-cellulose and cellobiose } \\
- \\
\text { Rice straw }\end{array}$ & $\begin{array}{c}\text { Cleavages } \beta-1,4 \text { linkages } \\
\text { of cellobiose }\end{array}$ & $\begin{array}{c}{[52]} \\
- \\
{[53]} \\
- \\
{[54]}\end{array}$ \\
\hline & 3.2.1.4 & $\begin{array}{l}\text { Endo- } \beta-1,4- \\
\text { glucanase } \\
\text {-Endoglucanase }\end{array}$ & $\begin{array}{c}\text { Cellulosilyticum lentocellum } \\
\text { Clostridium cellobioparum } \\
\text { Clostridium cellulolyticum } \\
\text { Eubacterium cellulosolvens } \\
\text { Clostridium } \\
\text { saccharoperbutylacetonicum } \\
- \\
\text { Clostridium cellulolyticum } \\
\text { Clostridium josui } \\
- \\
\text { Clostridium termitidi } \\
- \\
\text { Pantoea ananatis Sd-1 }\end{array}$ & $\begin{array}{c}\text { Corn stover } \\
\\
\\
\text { Filter paper } \\
- \\
\alpha \text {-cellulose and cellobiose } \\
- \\
\text { Rice straw }\end{array}$ & $\begin{array}{l}\text { Hydrolyzes } \beta-1,4 \text { bonds } \\
\text { in the amorphous } \\
\text { regions of cellulose }\end{array}$ & $\begin{array}{c}- \\
{[55]} \\
\\
- \\
{[53]} \\
- \\
{[54]}\end{array}$ \\
\hline
\end{tabular}


Table 1. Cont.

\begin{tabular}{|c|c|c|c|c|c|c|}
\hline & EC Number & Putative Function & Organism Source & Substrate & Activity or Function & References \\
\hline & 3.2.1.91 & $\begin{array}{l}\text { Cellobiohydrolase - } \\
\text { Exoglucanase - 1,4- } \\
\beta \text {-cellobiosidase }\end{array}$ & $\begin{array}{c}\text { Clostridium } \\
\text { saccharoperbutylacetonicum } \\
\text { Clostridium cellulyticum } \\
\text { Clostridium ruminicola } \\
- \\
\text { Clostridium termitidi } \\
- \\
\text { Clostridium josui } \\
- \\
\text { Pantoea ananatis Sd-1 } \\
- \\
\text { Caldicellulosiruptor bescii } \\
\text { Caldicellulosiruptor obsidiansis }\end{array}$ & $\begin{array}{c}\text { Corn stover } \\
- \\
\alpha \text {-cellulose and cellobiose } \\
- \\
\text { Filter paper } \\
- \\
\text { Rice straw } \\
- \\
\text { Crystalline cellulose }\end{array}$ & $\begin{array}{c}\text { Attack crystalline } \\
\text { cellulose in the } \beta-1,4 \\
\text { linkages }\end{array}$ & $\begin{array}{c}{[52]} \\
\\
- \\
{[53]} \\
- \\
- \\
{[55]} \\
- \\
{[54]} \\
- \\
{[56]}\end{array}$ \\
\hline & 3.2. 1.86 & $\begin{array}{l}\text { 6-phospho- } \beta \text { - } \\
\text { glucosidase }\end{array}$ & $\begin{array}{l}\text { Clostridium butyricum } \\
\text { Enterococcus casseliflavus }\end{array}$ & Corn stover & $\begin{array}{l}\text { Cleavage } \beta-1,4 \text {-linked } \\
\text { cellobiose 6-phosphate }\end{array}$ & [52] \\
\hline & 2.4.1.20 & $\begin{array}{c}\text { Cellobiose } \\
\text { phosphorylase }\end{array}$ & Clostridium phytofermentans & Corn stover & $\begin{array}{l}\text { Catalyzes the } \\
\text { reversible } \\
\text { phosphorolysis of } \\
\text { cellobiose }\end{array}$ & {$[52]$} \\
\hline & NA & Cellulase & $\begin{array}{c}\text { Clostridium cellobioparum } \\
\text { Clostridium lentocellum Clostridium } \\
\text { cellulolyticum } \\
- \\
\text { Caldicellulosiruptor bescii }\end{array}$ & $\begin{array}{c}\text { Filter paper } \\
- \\
\text { Crystalline cellulose }\end{array}$ & $\begin{array}{l}\text { Cleavage the } \beta-1,4 \\
\text { linkages in cellulose }\end{array}$ & $\begin{array}{c}{[55]} \\
- \\
{[56]}\end{array}$ \\
\hline & 3.2.1.1 & $\alpha$-amylase & $\begin{array}{c}\text { Clostridium } \\
\text { saccharoperbutylacetonicum } \\
- \\
\text { Caldicellulosiruptor bescii } \\
\text { Caldicellulosiruptor obsidiansis }\end{array}$ & $\begin{array}{c}\text { Corn stover } \\
- \\
\text { Crystalline cellulose }\end{array}$ & $\begin{array}{l}\text { Hydrolyze the } \\
\alpha-1,4 \text {-glucosidic bonds } \\
\text { in } \alpha \text {-glucans }\end{array}$ & $\begin{array}{c}{[52]} \\
- \\
{[56]}\end{array}$ \\
\hline & 3.2.1.39 & $\begin{array}{l}\text { Endo-1,3- } \beta- \\
\text { glucanase }\end{array}$ & Caldicellulosiruptor obsidiansis & Crystalline cellulose & $\begin{array}{l}\text { Hydrolyzes } \beta-1,3 \text {-bonds } \\
\text { present in glucans }\end{array}$ & [56] \\
\hline \multirow[t]{7}{*}{ Hemicellulose } & e 3.2 .1 .8 & $\begin{array}{l}\text { Xylanase- } \\
\text { Endoxylanase- } \\
\text { Endo- } \beta-1,4- \\
\text { xylanase }\end{array}$ & $\begin{array}{c}\text { Cellulosilyticum lentocellum } \\
\text { Roseburia intestinalis } \\
\text { Ruminococcus sp. } \\
\text { Cellulosilyticum ruminicola } \\
\text { Lachnoclostridium phytofermentans } \\
\text { Butyrivibrio fibrisolvens } \\
\text { Clostridium cellulosi } \\
- \\
\text { Clostridium termitidi } \\
- \\
\text { Caldicellulosiruptor bescii } \\
\text { Caldicellulosiruptor obsidiansis }\end{array}$ & $\begin{array}{c}\text { Corn stover } \\
\begin{array}{c}\text { Collulose } \\
- \\
\text { Crystalline cellulose }\end{array}\end{array}$ & $\begin{array}{l}\text { Attack } \beta-1,4 \text { bond of the } \\
\text { xylan backbone }\end{array}$ & $\begin{array}{c}- \\
{[53]} \\
- \\
{[56]}\end{array}$ \\
\hline & 3.2.1.23 & $\beta$-galactosidase & $\begin{array}{c}\text { Clostridium sp. } \\
- \\
\text { Caldicellulosiruptor bescii } \\
\text { Caldicellulosiruptor obsidiansis }\end{array}$ & $\begin{array}{c}\text { Corn stover } \\
- \\
\text { Crystalline cellulose }\end{array}$ & $\begin{array}{c}\text { Hydrolyze } \\
\beta-1,4 \text {-glycosidic linkage } \\
\text { present in lactose }\end{array}$ & $\begin{array}{c}{[52]} \\
- \\
{[56]}\end{array}$ \\
\hline & 3.2.1.89 & $\begin{array}{c}\text { arabinogalactan } \\
\text { endo-1,4- } \beta- \\
\text { galactosidase }\end{array}$ & $\begin{array}{c}\text { Paenibacillus sp. } \\
- \\
\text { Caldicellulosiruptor obsidiansis }\end{array}$ & Corn stover & $\begin{array}{l}\text { Hydrolyze } \beta-1,4 \\
\quad \text { linkages in } \\
\text { arabinogalactans }\end{array}$ & {$[52]$} \\
\hline & 3.2.1.25 & $\begin{array}{l}\text { Endo-1,4- } \beta \text { - } \\
\text { mannosidase }\end{array}$ & $\begin{array}{c}\text { Clostridium clariflavum } \\
- \\
\text { Caldicellulosiruptor bescii } \\
\text { Caldicellulosiruptor obsidiansis }\end{array}$ & $\begin{array}{c}\text { Corn stover } \\
- \\
\text { Crystalline cellulose }\end{array}$ & $\begin{array}{c}\text { Cleavage the } \\
\beta-1,4 \text {-manno-oligomers }\end{array}$ & $\begin{array}{c}{[52]} \\
- \\
{[56]}\end{array}$ \\
\hline & 3.2.1.131 & $\alpha$-glucuronidase & Paenibacillus sp. & Corn stover & $\begin{array}{c}\text { Hydrolyze } \\
\alpha-1,2 \text {-glycosidic linkage } \\
\text { between xylose and } \\
\text { glucuronic acid }\end{array}$ & [52] \\
\hline & 3.2.1.31 & $\beta$-glucuronidase & Clostridium cellulovorans & Corn stover & $\begin{array}{c}\text { Exohydrolyze } \\
\beta \text {-d-glucuronic acid } \\
\text { residues of } \\
\text { glycosaminoglycan }\end{array}$ & [52] \\
\hline & 3.2.1.37 & $\beta$-xylosidase & $\begin{array}{l}\text { Sphaerochaeta coccoides } \\
\text { Clostridium } \\
\text { saccharoperbutylacetonicum } \\
\text { Clostridium ruminicola } \\
\text { Flavobacterium johnsoniae } \\
\text { Cellulosilyticum ruminicola }\end{array}$ & Corn stover & $\begin{array}{l}\text { Exohydrolyze } \beta-1,4 \\
\text { linkages of xylans, to } \\
\text { removing xylose } \\
\text { residues }\end{array}$ & [52] \\
\hline
\end{tabular}


Table 1. Cont.

\begin{tabular}{|c|c|c|c|c|c|}
\hline EC Number & Putative Function & Organism Source & Substrate & Activity or Function & References \\
\hline 3.2.1.6 & $\begin{array}{l}\text { Endo -1,3(4)- } \beta-\alpha- \\
\quad \text { Glucanase }\end{array}$ & Clostridium perfringens & Corn stover & $\begin{array}{c}\text { Endohydrolysis of } \beta-1,3 \\
\text { or } \beta-1,4 \text { linkages in } \\
\beta \text {-D-glucans }\end{array}$ & [52] \\
\hline 3.2.1.78 & $\beta$-mannanase & $\begin{array}{c}\text { Clostridium clariflavum } \\
\text { Roseburia intestinalis } \\
\text { Cellulosilyticum lentocellum } \\
- \\
\text { Clostridium termitidi }\end{array}$ & $\begin{array}{l}\text { Corn stover } \\
\alpha \text {-cellulose }\end{array}$ & $\begin{array}{c}\text { Attack the } \beta-1,4 \text { bond in } \\
\text { D-mannan }\end{array}$ & $\begin{array}{l}{[52]} \\
{[53]}\end{array}$ \\
\hline 3.2.1.177 & $\alpha$-xylosidase & Paenibacillus mucilaginosus & Corn stover & $\begin{array}{l}\text { Hydrolyze } \alpha-1,6 \text { linked } \\
\text { xylose residues }\end{array}$ & [52] \\
\hline 3.2.1.55 & $\begin{array}{c}\alpha-\mathrm{L}- \\
\text { Arabinofuranosidase }\end{array}$ & $\begin{array}{c}\text {-Enterococcus casseliflavus } \\
\text { Enterococcus mundtii } \\
\text { Klebsiella pneumoniae } \\
- \\
\text { Clostridium termitidi } \\
- \\
\text { Thermobacillus xylanolyticus } \\
- \\
\text { Caldicellulosiruptor obsidiansis }\end{array}$ & 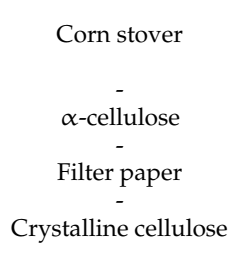 & $\begin{array}{c}\text { Exohydrolyze } \alpha \text {-L-1,5 } \\
\text { and/or } \alpha \text {-L-1,3 linkages } \\
\text { of } \\
\text { arabinofuranosyl-based } \\
\text { oligomers }\end{array}$ & $\begin{array}{c}{[52]} \\
- \\
- \\
{[53]} \\
- \\
{[55]} \\
- \\
{[56]}\end{array}$ \\
\hline 3.2.1.51 & $\alpha$-L-fucosidase & Caldicellulosiruptor obsidiansis & Crystalline cellulose & $\begin{array}{l}\text { Cleavage } \alpha-1,6-, \alpha-1,3-, \\
\alpha-1,4-, \text { and } / \text { or } \alpha-1,2 \\
\text { bonds in fucosylated } \\
\text { oligosaccharides }\end{array}$ & [56] \\
\hline 3.1.1.72 & acetylxylan esterase & $\begin{array}{c}\text { Enterococcus casseliflavus } \\
\text { Pseudobutyrivibrio xylanivorans } \\
- \\
\text { Clostridium termitidi }\end{array}$ & $\begin{array}{c}\text { Corn stover } \\
- \\
\alpha \text {-cellulose }\end{array}$ & $\begin{array}{l}\text { Remove the O-acetyl } \\
\text { groups from the O-2 } \\
\text { and/or O-3 positions }\end{array}$ & $\begin{array}{c}{[52]} \\
- \\
{[53]}\end{array}$ \\
\hline 3.1.1.1 & Carboxylesterase & Caldicellulosiruptor obsidiansis & Crystalline cellulose & $\begin{array}{l}\text { Hydrolyzes ester bonds, } \\
\text { liberating alcohol and } \\
\text { carboxylic acid }\end{array}$ & [56] \\
\hline NIA & Esterase & $\begin{array}{c}\text { Clostridium clariflavum } \\
\text { Clostridium josui } \\
- \\
\text { Clostrodium termitidi } \\
- \\
\text { Pantoea ananatis Sd-1 }\end{array}$ & $\begin{array}{c}\text { Corn stover } \\
- \\
\alpha \text {-cellulose } \\
- \\
\text { Rice straw }\end{array}$ & Cleavage ester bonds & $\begin{array}{c}{[52]} \\
- \\
{[53]} \\
- \\
{[54]}\end{array}$ \\
\hline 3.5.1.41 & Chitin deacetylase & Clostridium termitidi & Cellobiose & $\begin{array}{c}\text { Hydrolyze the } \\
\mathrm{N} \text {-acetoamido groups of } \\
\mathrm{N} \text {-acetyl- } \beta \text {-D- } \\
\text { glucosaminide in } \\
\text { chitin }\end{array}$ & [53] \\
\hline 3.2.1.14 & Chitinase & Clostridium termitidi & cellobiose & $\begin{array}{c}\text { Endo-hydrolyzes } \\
\mathrm{N} \text {-acetyl- } \beta \text {-D- } \\
\text { glucosaminide } \beta-1,4 \\
\text { linkages in chitin and } \\
\text { chitodextrins. }\end{array}$ & [53] \\
\hline 3.2.1.52 & $\begin{array}{c}\beta-\mathrm{N}- \\
\text { acetylhexosaminidase }\end{array}$ & Pantoea ananatis Sd-1 & Rice straw & $\begin{array}{c}\text { Hydrolyse the } \beta-1,4 \\
\text { glycosidic bond between } \\
\mathrm{N} \text {-acetylglucosamine } \\
\text { and anhydro-N- } \\
\text { acetylmuramic } \\
\text { acid }\end{array}$ & [54] \\
\hline NIA & $\begin{array}{l}\text { Cellulosomal } \\
\text { proteins }\end{array}$ & $\begin{array}{c}\text { Clostridium termitidi } \\
- \\
\text { Clostridium josui } \\
\text { Clostridium cellulolyticum }\end{array}$ & $\begin{array}{l}\alpha \text {-cellulose } \\
\text { Filter paper }\end{array}$ & $\begin{array}{l}\text { Protein complex that } \\
\text { achieves hydrolysis } \\
\text { cellulose and } \\
\text { hemicellulose }\end{array}$ & $\begin{array}{c}{[53]} \\
- \\
{[55]}\end{array}$ \\
\hline NIA & $\begin{array}{l}\text { Cellulosomal } \\
\text { xylanase }\end{array}$ & Clostridium cellulolyticum & Filter paper & $\begin{array}{c}\text { Hydrolyzes } \beta-1,4 \\
\text { linkages in the xylan } \\
\text { backbone }\end{array}$ & [55] \\
\hline
\end{tabular}

NIA: no information available. 
Table 2. List of cellulose and lignin degrading enzymes produced by different anaerobic bacteria.

\begin{tabular}{|c|c|c|c|c|}
\hline Microorganism & Substrate (Concentration) & Identified Enzymes & $\begin{array}{c}\text { Number of Different } \\
\text { Proteins }{ }^{\text {a }}\end{array}$ & Reference \\
\hline $\begin{array}{c}\text { Clostridium thermocellum } \\
\text { ATCC } 27405\end{array}$ & Avicel (2 g/L) & $\begin{array}{c}\text { Exoglucanase } \\
\text { Endoglucanase } \\
\text { Xylanase } \\
\text { Xyloglucanase } \\
\text { Lichenase } \\
\text { Mannanase } \\
\text { Chitinase } \\
\text { Endopygalactorunase } \\
\text { Glycosyl hydrolase }\end{array}$ & $\begin{array}{c}3 \\
11 \\
3 \\
1 \\
1 \\
1 \\
1 \\
1 \\
9\end{array}$ & [57] \\
\hline $\begin{array}{l}\text { Clostridium thermocellum } \\
\text { ATCC } 27405\end{array}$ & Cellobiose (2 g/L) & $\begin{array}{c}\text { Xylanase } \\
\text { Endoglucanase } \\
\text { Exoglucanase } \\
\text { Xyloglucanase } \\
\text { Chitinase } \\
\alpha \text {-l-arabinofuranosidase B } \\
\text { Glycoside hydrolase }\end{array}$ & $\begin{array}{l}5 \\
9 \\
3 \\
1 \\
1 \\
2 \\
9\end{array}$ & [57] \\
\hline Clostridium cellulovorans & Cellobiose (3 g/L) & $\begin{array}{c}\text { Endoglucanase } \\
\text { Mannanase } \\
\text { Exocellulase }\end{array}$ & $\begin{array}{l}5 \\
4 \\
1\end{array}$ & [58] \\
\hline Clostridium cellulovorans & Avicel (3 g/L) & $\begin{array}{l}\text { Endoglucanase } \\
\text { Mannanase } \\
\text { Xylanase } \\
\text { Exocellulase }\end{array}$ & $\begin{array}{l}6 \\
4 \\
1 \\
1\end{array}$ & [58] \\
\hline Clostridium cellulovorans & Xylan (3 g/L) & $\begin{array}{c}\text { Endoglucanase } \\
\text { Mannanase } \\
\text { Xylanase } \\
\text { Exocellulase }\end{array}$ & $\begin{array}{l}8 \\
4 \\
2 \\
1\end{array}$ & [58] \\
\hline Clostridium cellulolyticum $\mathrm{H} 10$ & $\begin{array}{l}\text { Washed hatched wheat straw } \\
\qquad(5 \mathrm{~g} / \mathrm{L})\end{array}$ & $\begin{array}{c}\text { Endoglucanase } \\
\text { Acetyl xylan esterase } \\
\text { Mannanase } \\
\text { Rhamnogalacturonan lyase } \\
\text { Xylanase } \\
\text { Cellobiohydrolase } \\
\text { Cellulase } \\
\text { Feruloyl esterase } \\
\text { Xyloglucanase } \\
\text { Arabinosidase } \\
\alpha \text {-arabinofuranosidase } \\
\alpha \text {-galactosidase } \\
\beta \text {-galactosidase }\end{array}$ & $\begin{array}{c}17 \\
2 \\
2 \\
1 \\
10 \\
3 \\
1 \\
2 \\
1 \\
1 \\
1 \\
2 \\
1\end{array}$ & [59] \\
\hline Pandoraea sp. ISTKB * & Kraft lignin (2 g/L) & $\begin{array}{c}\text { Peroxidases } \\
\text { Laccase } \\
\text { Oxidases } \\
\text { Oxidoreductases } \\
\text { Vanillate-O-demethylase } \\
\text { Dioxygenases } \\
\text { Oxygenases } \\
\text { Monooxygenase }\end{array}$ & $\begin{array}{c}4 \\
1 \\
10 \\
16 \\
2 \\
13 \\
2 \\
1\end{array}$ & {$[60]$} \\
\hline Aspergillus fumigatus G-13 ${ }^{*}$ & $\begin{array}{c}\mathrm{p} \text {-coumaric acid }(0.1 \\
\mathrm{mmol} / \mathrm{L}), \text { sinapic acid }(0.1 \\
\mathrm{mmol} / \mathrm{L}), \text { glucose }(10 \mathrm{~g} / \mathrm{L}) \\
\text { and cellulose }(10 \mathrm{~g} / \mathrm{L})\end{array}$ & $\begin{array}{c}\text { Dioxygenase } \\
\text { Glyoxylase } \\
\text { Oxidoreductase } \\
\text { Ferulic acid esterase } \\
\text { Monooxygenase } \\
\text { Catalase peroxidase } \\
\text { Cellulase } \\
\text { ß-glucancellobiohydrolase } \\
\text { Cellobiose dehydrogenase } \\
\text { Peroxidase } \\
\text { Methyltransferase } \\
\text { Oxidase } \\
\text { Ketoreductase } \\
\text { Aldo keto reductase } \\
\text { Catalase }\end{array}$ & $\begin{array}{l}8 \\
1 \\
5 \\
2 \\
8 \\
1 \\
1 \\
1 \\
1 \\
1 \\
2 \\
1 \\
1 \\
1 \\
2\end{array}$ & {$[61]$} \\
\hline Phanerochaete chrysosporium ${ }^{*}$ & $\begin{array}{l}\text { Softwood ( } 30 \mathrm{~g} \text { with } 75 \% \\
\text { moisture content) }\end{array}$ & $\begin{array}{c}\beta \text {-Glucosidase } \\
\text { Mannanase } \\
\text { Endoglucanase } \\
\text { Exocellobiohydrolase } \\
\text { Mannosidase } \\
\text { Oxidase } \\
\text { Lignin peroxidase }\end{array}$ & $\begin{array}{l}3 \\
1 \\
2 \\
3 \\
1 \\
1 \\
1\end{array}$ & [62] \\
\hline
\end{tabular}

${ }^{a}$ Number of identified proteins showing corresponding enzyme activity. ${ }^{*}$ Aerobic microorganisms. 
$\beta$-xylosidases (EC 3.2.1.37) act upon the $\beta-1,4$ bonds on the nonreducing ends of xylooligomers, xylobiose and, in some cases, on xylan by employing a retaining mechanism. Meanwhile, $\alpha$-L-arabinofuranosidases (EC 3.2.1.55) are arabinases that hydrolyze $\alpha$-L-arabinofuranosyl groups acting on $\alpha-\mathrm{L}-1,3$ and $\alpha$-L-1,5 linkages of arabinans, arabinoxylans and arabinogalactans. Enzymes $\alpha$-L-arabinofuranosidases use retaining or inverting mechanisms. Meanwhile, $\beta$-mannanases (EC 3.2.1.78) and $\beta$-mannosidases (EC 3.2.1.25) act sequentially, $\beta$-mannanases hydrolyze mannan-based saccharides to produce $\beta$-1,4-manno-oligomers, which are then hydrolyzed by $\beta$-mannosidases to yield mannose. Finally, $\alpha$-glucuronidases (EC 3.2.1.131) [43,63] hydrolyze $\alpha-1,2$ linkages between xylose and D-glucuronic acid by inverting mechanism. Clostridium spp. encode most of these hemicellulolytic enzymes (Table 1) and has shown a great potential for genetic engineering to improve lignocellulose hydrolysis.

Hemicellulolytic carbohydrate esterases remove the ester group from carbohydrates and facilitates the access to GHs [64]. Esterases are classified into 18 families according to the CAZy database (www.CAZy.org; accessed on 23 September 2021), these include feruloyl esterases (EC 3.1.1.73) from the CE1 family which catalyze the cleavage of the ester bond at the O-5 position between a ferulic acid and arabinose liberating hydroxycinnamic acids; as well as acetyl xylan esterases (EC 3.1.1.72), which in turn catalyzes the cleavage of ester linkages on the position O-2 and O-3 between an acetyl group and xylose [45,65]. Various studies reported higher expression of hemicellulases than cellulases, as hemicellulose is more exposed than cellulose [52,53]. In general, the polysaccharides are hydrolyzed either by extracellular enzymes and/ or by the cellulosome (Figure 2A).

\subsection{Enzymes Involved in Lignin Degradation}

Lignin is one of the most complex substrates compared to the other components of the lignocellulosic structure (cellulose and hemicellulose), and its depolymerization involves a variety of enzymes (Table 3). Under aerobic conditions, the main degradative enzymes are peroxidases and laccases, which need molecular oxygen for their catalytic activity [66]. On the contrary, several enzymes participate in lignin degradation under anaerobic conditions. Auxiliary Activities (AAs) are a recent family of catalytic proteins in the CAZy database, which are redox enzymes and are classified into 16 subfamilies, including different peroxidases (like lignin peroxidase and manganese peroxidase) and laccases, which are known to act on lignin. Other enzymes in this classification include oxidases, demethylases, and reductases [67].

Table 3. Enzymes involved in different pathways of lignin degradation.

\begin{tabular}{|c|c|c|c|c|}
\hline Reaction/Pathway & Enzyme & Microorganism & Gene & Reference \\
\hline \multirow[t]{3}{*}{$\beta-\mathrm{O}-4$ aryl ether } & $\mathrm{C} \alpha$-dehydrogenase & Sphingobium sp. SYK -6 & $\begin{array}{l}\text { ligD } \\
\text { ligL } \\
\text { ligN } \\
\text { ligO }\end{array}$ & {$[68,69]$} \\
\hline & $\beta$-etherase & Sphingobium sp. SYK -6 & $\begin{array}{l}\text { ligF; } \operatorname{lig} E \\
\operatorname{lig} P\end{array}$ & {$[68,69]$} \\
\hline & Glutathione lyase & Sphingobium sp. SYK -6 & $\operatorname{lig} G$ & [68] \\
\hline \multirow[t]{2}{*}{ O-demethylation } & Syringate-O-demethylase & Sphingobium sp. SYK -6 & $\operatorname{des} A$ & [70] \\
\hline & Vanillate O-demethylase & Sphingobium sp. SYK -6 & $\operatorname{lig} M$ & [70] \\
\hline \multirow[t]{5}{*}{ Benzoyl-CoA pathway } & Ligase & Rhodopseudomonas palustris & $h b a A$ & [71] \\
\hline & Reductase & Thauera aromatica & NIA & [72] \\
\hline & pHB-CoA reductase & Rhodopseudomonas palustris & $h b a B C D$ & [71] \\
\hline & Benzoyl-CoA reductase class 1 & Thauera aromatica & $\begin{array}{l}b c r A \\
b c r D \\
b c r B \\
b c r C\end{array}$ & [73] \\
\hline & Benzoyl-CoA reductase class 2 & Geobacter metallireducens & $\begin{array}{c}\text { bamB } \\
\text { bamC } \\
\text { bamDE } \\
\text { bamCF } \\
\text { bam } G H I\end{array}$ & {$[74,75]$} \\
\hline
\end{tabular}


Table 3. Cont.

\begin{tabular}{|c|c|c|c|c|}
\hline Reaction/Pathway & Enzyme & Microorganism & Gene & Reference \\
\hline & Cyclohexadienoyl-CoA hydratase & Geobacter metallireducens & bamR & {$[74]$} \\
\hline & Hydroxyenoyl-CoA dehydrogenase & Geobacter metallireducens & bamQ & [74] \\
\hline & oxoacyll-CoA hydrolase & Geobacter metallireducens & bamA & [74] \\
\hline \multirow[t]{6}{*}{$\begin{array}{c}\beta \text {-oxidation- Benzoyl-CoA } \\
\text { pathway }\end{array}$} & Hydroxyacyl-CoA dehydrogenase & Geobacter metallireducens & $\operatorname{pim} E$ & [75] \\
\hline & $\begin{array}{c}\text { Acyl-CoA acetyltransferase } \\
\text { ( } \beta \text {-Ketothiolase })\end{array}$ & Geobacter metallireducens & $\operatorname{pim} B$ & {$[75]$} \\
\hline & Glutaryl-CoA dehydrogenase & Geobacter metallireducens & $g c d H$ & [75] \\
\hline & 3-hydroxybutyryl-CoA dehydratase & Geobacter metallireducens & NIA & [75] \\
\hline & $\begin{array}{l}\text { 3-Hydroyibutyryl-CoA } \\
\text { dehydrogenase }\end{array}$ & Geobacter metallireducens & NIA & [75] \\
\hline & Acetoacetyl-CoA thiolase & Geobacter metallireducens & NIA & {$[75]$} \\
\hline \multirow[t]{6}{*}{$\beta$-Ketoadipate pathway } & Protocatechuate 3,4-dioxygenase & Pseudomonas putida & pcaGH & [76] \\
\hline & Cycloisomerase & Pseudomonas putida & $p c a B$ & {$[76]$} \\
\hline & $\begin{array}{c}\gamma \text {-Carboxy-muconolactone } \\
\text { decarboxylase }\end{array}$ & Pseudomonas putida & $p c a C$ & [76] \\
\hline & $\begin{array}{l}\beta \text {-ketoadipate enol-lactone } \\
\text { hydrolase }\end{array}$ & Pseudomonas putida & pacD & {$[76]$} \\
\hline & $\begin{array}{c}\beta \text {-ketoadipate succinyl-CoA } \\
\text { transferase }\end{array}$ & Pseudomonas putida & pcalJ & [76] \\
\hline & $\beta$-ketoadipate-CoA thiolase & Pseudomonas putida & pcaF & [76] \\
\hline \multirow[t]{4}{*}{ Phloroglucinol pathway } & Gallate decarboxylase & Lactobacillus plantarum & $\begin{array}{l}\operatorname{lpdB} \\
\operatorname{lpdC} \\
l p d D\end{array}$ & [77] \\
\hline & Pyrogallol transhydroxylase & Pelobacter acidigallici & $\begin{array}{l}\text { athL } \\
\text { bthL }\end{array}$ & [78] \\
\hline & Phloroglucinol reductase & Pelobacter acidigallici & NIA & [79] \\
\hline & Dihydrophloroglucinol hydrolase & Pelobacter acidigallici & NIA & [79] \\
\hline \multirow[t]{6}{*}{$\begin{array}{c}\beta \text {-oxidation- Phloroglucinol } \\
\text { pathway }\end{array}$} & 3-hydroxyacyl-CoA dehydrogenase & Pelobacter acidigallici & NIA & [79] \\
\hline & Acetyl CoA transferase & Pelobacter acidigallici & NIA & {$[79]$} \\
\hline & Triacetic acid $\beta$-ketothiolase & Pelobacter acidigallici & NIA & [79] \\
\hline & Acetoacetyl-CoA $\beta$-ketothiolase & Pelobacter acidigallici & NIA & [79] \\
\hline & Phosphotransacetylase & Pelobacter acidigallici & NIA & {$[79]$} \\
\hline & Acetate kinase & Pelobacter acidigallici & NIA & [79] \\
\hline
\end{tabular}

NIA: no information available.

Lignin is an aromatic heteropolymer of phenylpropanes (mainly coniferyl, p-coumaryl, and sinapyl alcohols) bound to hemicellulose and cellulose and intermolecularly connected by carbon-carbon and aryl-ether linkages [40,73]. $\beta$-O-4 aryl ether bonds are the most predominant intermolecular bonds present on lignin which represent the $45-60 \%$ of the total linkages [69]. Three main enzymes, viz., $\mathrm{C} \alpha$-dehydrogenase, $\beta$-etherase and glutathione lyase are implicated in the breakdown of the $\beta-\mathrm{O}-4$ aryl ether bonds. $\mathrm{C} \alpha$-dehydrogenase is a NAD ${ }^{+}$dependent enzyme that oxidizes benzyl alcohol at $\mathrm{C} \alpha$ position increasing the polarity, which facilitates the $\beta$-etherase activity. The glutathione dependent $\beta$-etherase breaks the ether bond by the addition of glutathione that is later eliminated by glutathione lyases [68]. These three intracellular enzymes cannot act on high molecular weight lignin. Otsuka et al. [80] reported a $\beta$-etherase that does not need glutathione and uses molecules of water to cleavage at $C \alpha$ and $C \beta$ positions of the $\beta-O-4$ aryl ether bonds extracellularly [81]. Lignin depolymerization results in different lignin derivatives, and the most common are vainillate and syringate. Subsequently, demethylation of vainillate and syringate by vainillate $\mathrm{O}$-demethylase and syringate $\mathrm{O}$-demethylase, respectively, are important steps to produce protocatechuate and gallate, which as intermediaries enter different pathways for ring cleavage [70,82]. Under anaerobic conditions, protocatechuate and gallate are converted to different key intermediates such as benzoyl-CoA, phloroglucinol, hydroxy- 
hydroquinone and resorcinol [73]. Benzoyl-CoA, being the most common intermediate, is used as biomarker in anaerobic degradation of aromatic compounds [83]. A schematic diagram of the main reactions involved in anaerobic lignin degradation, key intermediates and ring cleavage is shown in Figure 2B. Protocatechuate anaerobic degradation can be via the benzoyl-CoA pathway or $\beta$-ketoadipate pathway, whereas gallate anaerobic degradation takes place by the phloroglucinol pathway [82]. The benzoyl-CoA pathway consists of four main steps, which are (i) activation, (ii) ring reduction, (iii) ring cleavage and (iv) conversion to acetyl-CoA $[71,74]$. The participating enzymes of this pathway are summarized in Table 3. The $\beta$-ketoadipate pathway is a conserved metabolic route that starts with the protocatechuate ring cleavage by protocatechuate 3,4-dioxygenase resulting in $\beta$-carboxymuconate, which passes through several reactions to form $\beta$-ketoadipate, which is then ligated to a coenzyme A by a transferase and finally separated into succinyl-CoA and acetyl-CoA [84].

Phloroglucinol is an intermediate found during gallate anaerobic degradation. Gallate initially undergoes decarboxylation by gallate decarboxylase forming pyrogallol, which is then converted to phloroglucinol by the transfer of a hydroxyl group. Then the phloroglucinol ring is cleaved by a hydrolase and the resulting product undergoes $\beta$-oxidation to obtain acetyl-CoA [78,79]. Table 3 enlists important enzymes of different pathways that target lignin or its derivatives in anaerobic conditions; the genes that encode these enzymes are also indicated. Although there are no complete proteomic analyzes on anaerobic ligninolytic enzymes, genomic and transcriptomic analyses have been employed for their identification $[82,85,86]$.

\section{Biofuel Production from Lignocellulosic Biomass}

The interest in the production of biofuels from renewable sources has increased in the recent years due to environmental concerns and the concomitant need to decrease our dependence on fossil-based energy resources [1]. Among the potential substrates, lignocellulose is a major renewable source with potential for application in various bioprocesses for production of value-added products [87]. Microbial production of different biofuels such as ethanol, methane, hydrogen, butanol and others using lignocellulosic residues as carbon source is presented in Table 4 .

Table 4. Reports on biofuels production using lignocellulosic biomass as feedstock.

\begin{tabular}{|c|c|c|c|c|c|c|c|}
\hline $\begin{array}{l}\text { Lignocellulosic } \\
\text { Feedstock }\end{array}$ & Feedstock Preparation & Biofuel & Inoculum & $\begin{array}{l}\text { Fermentation } \\
\text { Method }\end{array}$ & Biofuel Yield & Biofuel Titer & Reference \\
\hline Rice straw & $\begin{array}{l}\text { Alkaline pretreatment and } \\
\text { enzymatic hydrolysis }\end{array}$ & Biobutanol & $\begin{array}{l}\text { Clostridium } \\
\text { acetobutylicum } \\
\text { ATCC } 824\end{array}$ & $\begin{array}{c}\text { PVA- } \\
\text { immobilized }\end{array}$ & $0.23 \mathrm{~g} / \mathrm{g}$ glucose & $13.8 \mathrm{~g} / \mathrm{L}$ & [88] \\
\hline $\begin{array}{l}\text { Sugarcane } \\
\text { bagasse }\end{array}$ & $\begin{array}{l}\text { Alkaline pretreatment and } \\
\text { enzymatic hydrolysis }\end{array}$ & Biobutanol & $\begin{array}{l}\text { Clostridium } \\
\text { acetobutylicum } \\
\text { ATCC } 824\end{array}$ & Suspended cell & $0.16 \mathrm{~g} / \mathrm{g}$ glucose & $8.4 \mathrm{~g} / \mathrm{L}$ & [88] \\
\hline Rice straw & $\begin{array}{l}\text { Alkaline and acid } \\
\text { pretreatments and } \\
\text { enzymatic hydrolysis }\end{array}$ & Biobutanol & $\begin{array}{l}\text { Clostridium } \\
\text { beijerinckii F-6 }\end{array}$ & $\mathrm{ABE}$ & $0.13 \mathrm{~g} / \mathrm{g}$ & $4.22 \mathrm{~g} / \mathrm{L}$ & [89] \\
\hline Rice straw & $\begin{array}{l}\text { Mechanic, thermal, and } \\
\text { acid pretreatment }\end{array}$ & Biobutanol & $\begin{array}{l}\text { Clostridium } \\
\text { acetobutylicum } \\
\text { NCIM } 2337\end{array}$ & Batch & $0.34 \mathrm{~g} / \mathrm{g}$ & $13.5 \mathrm{~g} / \mathrm{L}$ & [90] \\
\hline $\begin{array}{l}\text { Sugarcane } \\
\text { bagasse }\end{array}$ & $\begin{array}{l}\text { Acid pretreatment and } \\
\text { enzymatic hydrolysis }\end{array}$ & Ethanol & $\begin{array}{l}\text { Saccharomyces } \\
\text { cerevisiae } \\
\text { XUSAE57 }\end{array}$ & NIA & $0.49 \mathrm{~g} / \mathrm{g}$ & NIA & [91] \\
\hline Oat spelt & Enzymatic hydrolysis & Ethanol & $\begin{array}{l}\text { Debaryomyces } \\
\text { hansenii }\end{array}$ & Immobilized & $0.46 \mathrm{~g} / \mathrm{g}$ & $8.38 \mathrm{~g} / \mathrm{L}$ & [92] \\
\hline Wheat bran & Enzymatic hydrolysis & Ethanol & $\begin{array}{l}\text { Debaryomyces } \\
\text { hansenii }\end{array}$ & Immobilized & $0.44 \mathrm{~g} / \mathrm{g}$ & $6.89 \mathrm{~g} / \mathrm{L}$ & [92] \\
\hline $\begin{array}{l}\text { Sugarcane } \\
\text { bagasse }\end{array}$ & $\begin{array}{l}\text { Alkaline pretreatment and } \\
\text { enzymatic hydrolysis }\end{array}$ & Ethanol & $\begin{array}{l}\text { Dekkera } \\
\text { bruxellensis } \\
\text { GDB248 }\end{array}$ & $\begin{array}{c}\text { Anaerobic } \\
\text { fermentation }\end{array}$ & $0.42 \mathrm{~g} / \mathrm{g}$ & $4.5 \mathrm{~g} / \mathrm{g}$ & [93] \\
\hline $\begin{array}{l}\text { Sweet sorghum } \\
\text { bagasse }\end{array}$ & $\begin{array}{l}\text { Alkaline pretreatment and } \\
\text { enzymatic hydrolysis }\end{array}$ & Ethanol & $\begin{array}{l}\text { Dekkera } \\
\text { bruxellensis } \\
\text { GDB248 }\end{array}$ & $\begin{array}{l}\text { Anaerobic } \\
\text { fermentation }\end{array}$ & $0.44 \mathrm{~g} / \mathrm{g}$ & $4.85 \mathrm{~g} / \mathrm{g}$ & [93] \\
\hline
\end{tabular}


Table 4. Cont.

\begin{tabular}{|c|c|c|c|c|c|c|c|}
\hline $\begin{array}{l}\text { Lignocellulosic } \\
\text { Feedstock }\end{array}$ & Feedstock Preparation & Biofuel & Inoculum & $\begin{array}{l}\text { Fermentation } \\
\text { Method }\end{array}$ & Biofuel Yield & Biofuel Titer & Reference \\
\hline $\begin{array}{l}\text { Bagasse, rice } \\
\text { straw, corncob }\end{array}$ & Acid pretreatment & Biogas & $\begin{array}{c}\text { Granular } \\
\text { anaerobic sludge } \\
\text { from chemical } \\
\text { plant }\end{array}$ & $\begin{array}{l}\text { Continuous } \\
\text { anaerobic } \\
\text { digestion }\end{array}$ & $\begin{array}{c}0.381 \mathrm{~L} / \mathrm{g} \mathrm{COD} \\
\left(69.6 \% \mathrm{CH}_{4}\right)\end{array}$ & NIA & [94] \\
\hline $\begin{array}{l}\text { A. tequilana } \\
\text { bagasse }\end{array}$ & Acid pretreatment & Methane & $\begin{array}{c}\text { Granular } \\
\text { anaerobic sludge } \\
\text { from full-scale } \\
\text { reactor }\end{array}$ & $\begin{array}{c}\text { Batch anaerobic } \\
\text { digestion }\end{array}$ & $\begin{array}{c}0.26 \mathrm{~L} \mathrm{CH}_{4} / \mathrm{g} \\
\mathrm{COD}\end{array}$ & NIA & [95] \\
\hline $\begin{array}{l}\text { Cornstalks } \\
\text { fermentation } \\
\text { effluents }\end{array}$ & Alkaline pretreatment & Methane & $\begin{array}{l}\text { Anaerobic } \\
\text { sludge }\end{array}$ & Batch & $\begin{array}{l}0.178 \mathrm{~L} \mathrm{CH}_{4} / \mathrm{g} \\
\text { cornstalks }\end{array}$ & NIA & [96] \\
\hline Cornstalks & Alkaline pretreatment & Hydrogen & $\begin{array}{l}\text { Clostridium } \\
\text { thermocellum } \\
7072\end{array}$ & $\begin{array}{l}\text { Two-stage batch } \\
\text { fermentation }\end{array}$ & $\begin{array}{l}0.074 \mathrm{~L} / \mathrm{g} \\
\text { cornstalks }\end{array}$ & NIA & [96] \\
\hline Cornstalks & Acid pretreatment & Hydrogen & $\begin{array}{l}\text { Microbial } \\
\text { consortium form } \\
\text { cow dung } \\
\text { compost }\end{array}$ & Batch & $\begin{array}{c}0.149 \mathrm{~L} \mathrm{H}_{2} / g \\
\text { TVS }\end{array}$ & NIA & [97] \\
\hline Wheat straw & Acid pretreatment & Hydrogen & $\begin{array}{l}\text { Microbial } \\
\text { consortium form } \\
\text { cow dung } \\
\text { compost }\end{array}$ & Batch & $0.068 \mathrm{~L} \mathrm{H}_{2} \mathrm{~g}$ TVS & NIA & [98] \\
\hline
\end{tabular}

NIA: no information available. ABE: acetone, butanol, ethanol. PVA: polyvinyl alcohol. COD: chemical oxygen demand.

Different strategies such as chemical pretreatments or/and enzymatic hydrolysis have been employed to recover the energy from lignocellulosic biomass (Table 4). However, biofuel yield varies widely depending on the choice of pretreatment and the microorganism employed. In general, chemical and mixed pretreatments achieved significant hydrolysis of hemicellulose and soluble lignin (Table 4). However, the formation of various inhibitory substances during chemical pretreatments limits recovery and yield [99]. Conversely, enzymatic hydrolysis is gaining attention to overcome inhibitory substances and thus improve yield. More information at the molecular level is needed to devise novel strategies to increase biofuel recovery. In this regard, proteomic analysis may reveal which enzymes are missing/present at distinct steps of treatment, thereby permitting a snapshot of the microbial activity, their metabolism and protein production. This in turn could favor the identification of biomarkers for optimizing and monitoring of the bioprocess.

\subsection{Proteomics of Ethanol Production}

Lignocellulosic biomass has been widely used for industrial production of bioethanol $[100,101]$. In general, the lignocellulosic biomass undergoes pretreatment for liberation of sugars, which are fermented to bioethanol. The interaction between cellulose hydrolytic enzymes is necessary for cellulose hydrolysis before the fermentation process starts. Based on the proteome analyses of several studies, there are some key proteins during ethanol production (Table 5). For example, alcohol dehydrogenase, acetaldehyde$\mathrm{CoA} /$ alcohol dehydrogenase, pyruvate formate lyase and glyceraldehyde-3-phosphate dehydrogenase. 
Table 5. Key enzymes up- and downregulated under different growth conditions in central carbon metabolism, pyruvate metabolism and ethanol production.

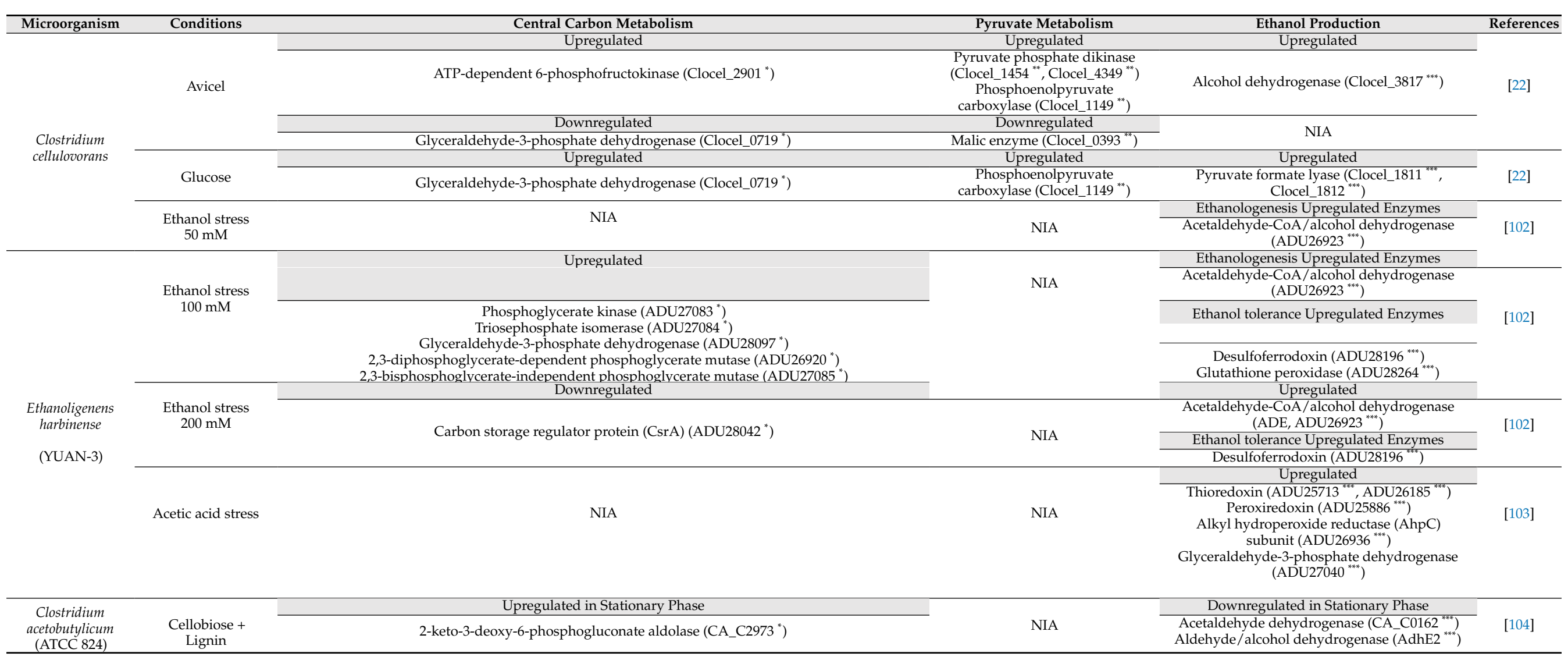


Table 5. Cont.

\begin{tabular}{|c|c|c|c|c|c|}
\hline Microorganism & Conditions & Central Carbon Metabolism & Pyruvate Metabolism & Ethanol Production & References \\
\hline \multirow{13}{*}{$\begin{array}{l}\begin{array}{c}\text { Caldicellulosiruptor } \\
\text { bescii }\end{array} \\
\text { (DSM 6725) }\end{array}$} & \multirow{8}{*}{$\begin{array}{c}\text { C5 substrates } \\
\text { (xylose and xylan) }\end{array}$} & Upregulated in xylan & \multirow{8}{*}{ NIA } & \multirow{8}{*}{ NIA } & \multirow{8}{*}{ [105] } \\
\hline & & Extracellular solute binding proteins (ESBP) & & & \\
\hline & & $\begin{array}{l}(\text { Athe_0849*) } \\
\left(\text { Athe } 0089^{*}\right)\end{array}$ & & & \\
\hline & & Upregulated in xylose and xylan & & & \\
\hline & & ESBPs & & & \\
\hline & & (Athe_0523*) & & & \\
\hline & & (Athe_2091*') & & & \\
\hline & & $\begin{array}{l}\left(\text { Athe_2574* }{ }^{*}\right) \\
\left(\text { Athe_0847 }{ }^{*}\right)\end{array}$ & & & \\
\hline & \multirow{5}{*}{$\begin{array}{c}\text { C6 substrates } \\
\text { (glucose, cellobiose } \\
\text { and avicel) }\end{array}$} & Upregulated in avicel & \multirow{5}{*}{ NIA } & \multirow{5}{*}{ NIA } & \multirow{5}{*}{ [105] } \\
\hline & & Glycoside hydrolases (Athe_0459") & & & \\
\hline & & (Athe_0460*) & & & \\
\hline & & Upregulated in glucose, cellobiose and avicel & & & \\
\hline & & $\begin{array}{c}\text { Xylose isomerase (Athe_0345 }{ }^{*} \text { ) } \\
\text { ABC transporter-related proteins (Athe_1109 }{ }^{*} \text { ) }\end{array}$ & & & \\
\hline
\end{tabular}

NIA: no information available. ${ }^{*}$ Central carbon metabolism; ${ }^{* *}$ pyruvate metabolism; ${ }^{* * *}$ ethanol production. 
According to Usai et al. [22], the cellulolytic bacterium Clostridium cellulovorans showed different kinetics and energetics based on the substrate that is used. This study showed global changes in C. cellulovorans proteome when grown on crystalline cellulose (avicel) and a soluble carbohydrate (glucose). Notably, ATP-dependent 6-phosphofructokinase, the principal regulatory enzyme for glycolysis pathway was upregulated when C. cellulovorans grew on avicel [106].

Moreover, Usai et al. [22] identified pyruvate phosphate dikinase (PPDK) as a putative key enzyme in the regulation of carbon flux during cellulose metabolism. They also reported that phosphoenolpyruvate carboxylase (PEPC), found in similar amounts in avicel and glucose, could replace the phosphoenolpyruvate carboxykinase (PEPCK) activity in the malate shunt, an alternative pathway for the conversion of phosphoenolpyruvate (PEP) to oxaloacetate. In addition, few alcohol dehydrogenases were upregulated in avicel, of which Clocel_3817 (an iron-containing alcohol dehydrogenase), was the most highly produced. The authors concluded that Clocel_3817 was possibly involved in the reduction of acetylcoA to acetaldehyde initially, and later to ethanol, concomitantly oxidizing two NADH to NAD. Conversely, a malic enzyme and a glyceraldehyde-3-phosphate dehydrogenase (GAPDH) were downregulated in the presence of avicel. On the contrary, GAPDH, an enzyme associated with bottlenecks in glycolysis pathway, showed upregulation in $C$. cellulovorans with glucose as substrate [22]. There were also three upregulated enzymes, glutamate dehydrogenase, glutamine synthetase and glutamate synthase, which are involved in nitrogen assimilation and synthesis of components of cell biomass. The activity of these enzymes plays a critical role in nitrogen assimilation and are present in most bacterial species.

Poudel et al. [105] reported the proteome of Caldicellulosiruptor bescii DSM6725. They analyzed the production of extracellular proteins across C5 (xylose and xylan) and C6 (glucose, cellobiose, avicel) substrate classes. Extracellular solute binding proteins (ESBPs) (enzymes that show response to a specific type of substrate) have non-catalytic extracellular activities and are important for lignocellulose deconstruction. Some ESBPs were found to be upregulated with $\mathrm{C} 5$ substrates such as the extracellular solute binding protein Athe_0089, an endo-1,4-beta-xylanase which was specific to xylan.

Other extracellular binding proteins (Athe_0523 and Athe_2091), specific to xylose and xylan and related to the hydrolysis of O-glycosyl compounds, were upregulated as well. Unlike C5 substrates, no extracellular solute binding proteins showed upregulation with C6 substrates. The study only recorded the activity of enzymes involved in the synthesis and breakdown of complex polysaccharides [30]. A pair of CAZymes belonging to the $\mathrm{GH}$ family were more abundant with avicel than cellobiose alone, despite their cellobiose/cellodextrin phosphorylase activities. Some other enzymes, such as xylose isomerase and $\mathrm{ABC}$ transporter-related proteins, were also upregulated with $\mathrm{C} 6$ substrates, indicating the importance of glucose transport. $A B C$ transporters are a group of proteins found in the membrane that transport solute molecules via the consumption of ATP [107]. Recently Zurawski et al. [108] reported that these transporters in coordination with CAZymes play an important role in enhancing the usage of the carbohydrate content of plant biomass by Caldicellulosiruptor species.

While substrate differences could affect production of enzymes, other parameters that such as presence of other molecules also affect enzymes. For instance, exogenous ethanol or acetic acid addition. Microbial ethanol stress response has generally been described to be a complex biological process. The molecular response to ethanol stress of Ethanoligenens harbinense strain $\mathrm{YUAN}-3$, an anaerobic bacterium capable of producing ethanol, acetic acid, hydrogen and $\mathrm{CO}_{2}$ was evaluated earlier [102]. They studied the protein production under different ethanol concentrations and reported that the bifunctional acetaldehyde$\mathrm{CoA} /$ alcohol dehydrogenase (ADHE) which generates ethanol from acetyl-CoA plays a key role in ethanol production [109], and showed upregulation at all tested concentrations of ethanol. They observed that ADHE production level was closely related to the endogenous ethanol yield, indicating that ethanol yield increases when exogenous ethanol is added to 
the medium. In addition, glycolysis related enzymes such as glyceraldehyde-3-phosphate dehydrogenase showed an upregulation at $100 \mathrm{mM}$ ethanol, demonstrating that this condition increases the demand for energy to increase tolerance. Some enzymes involved in ethanol-tolerance stress were also upregulated, for instance, desulfoferrodoxin and glutathione peroxidase, which protect organisms from oxidative stress. Urea carboxylase, allophanate hydrolase and two urea carboxylase-associated proteins were also upregulated during nitrogen metabolism at stress conditions of $50 \mathrm{mM}$ ethanol.

Likewise, acetic acid stress response was evaluated in E. harbinense YUAN-3, and upregulation of glyceraldehyde-3-phosphate dehydrogenase (ADU27040) was observed in the presence of $200 \mathrm{mM}$ acetic acid. This enzyme was recently related with the efficient repair of cytotoxic DNA lesions in E. coli, and Li et al. [103] suggested its possible role as a response to maintain DNA structure during acetic acid stress.

Phenolic compounds are the main inhibitor of acetone-butanol-ethanol fermentation in Clostridia. In a study by Raut et al. [104], the effect of lignin on cellobiose consumption by Clostridium acetobutylicum ATCC 824 was evaluated. Glycolysis, fermentation and associated pathways were significantly repressed in the presence of lignin, this was seen by the downregulation of some enzymes related to solvent production such as acetaldehyde dehydrogenase (CA_C0162) and an aldehyde/alcohol dehydrogenase (Adhe2), in which production has been suggested to be sensitive to culture conditions [110].

\subsection{Proteomics in the Production of Acids and Solvents}

Acid-solvent biosynthesis takes place through central carbon metabolism from different sources (glucose and xylose), and their key modulations (redox and energy generation) are well studied in the Clostridium genus [111]. When the substrates are metabolized through the central carbon pathways (glycolysis or pentose-phosphate) under anaerobic conditions, microorganisms produce acids from the main intermediary acetyl-CoA (Figure 3). Glyceraldehyde-3-phosphate dehydrogenase is the key enzyme as it generates NADH via glyceraldehyde-3-phosphate oxidation. This has been identified as a bottleneck of sugar metabolism for efficient acid-solvent production [22].

Anaerobes mostly oxidize pyruvate to acetyl-CoA via pyruvate ferredoxin oxidoreductase, which cleaves the carbon-carbon bonds for electron transfer coupling flavoproteins in the reduction of crotonyl-CoA to butyryl-CoA [112]. Acid formation begins from the CoA precursors (acetyl-CoA, crotonyl-CoA), which act to activate expression of genes that produce different enzymes of an organized operon. The activation of this operon for acid formation will depend on the environmental conditions (mainly $\mathrm{pH}$ ) and the energy requirements from the organism. The phosphate acetyltransferase (pta) and acetate kinase (ack) are strongly related to acid formation as the first step. Moreover, high levels of thiolase A (thlA), crotonase (crt,) and butyryl-CoA dehydrogenase (bcd) were reported to be mainly involved in acetyl-CoA to butyryl-CoA conversion. However, earlier studies indicated that the proteins CAP0036 and CAP0037 in Clostridium acetobutylicum, also regulate metabolism under acidogenic conditions [113,114]. Furthermore, under stressful acidic conditions ( $\mathrm{pH}$ 4.5), fermentation products such as acetate and butyrate and high levels of cofactors such as ATP, $\mathrm{NAD}(\mathrm{P}) \mathrm{H} / \mathrm{NAD}(\mathrm{P})^{+}$serve as signals that trigger a rapid shift in the metabolic pathways from acidogenesis to solventogenesis [115]. Other environmental conditions (temperature, digestion time) along with several stress-inducing compounds such as butyryl-phosphate and formic acid, may also alter cellular activities causing a shift of metabolism from acids production to solventogenesis.

This shift is controlled principally by three enzymes, acetoacetate decarboxylase (Adc), aldehyde/alcohol dehydrogenase (AdhE) and the acetoacetyl-CoA:acyl CoA transferase $(\mathrm{CtfA} / \mathrm{B})$, which at the start of the process, are downregulated during acid production but highly expressed during the production of solvents. During this shift, CoA-transferase $(\mathrm{Ctf} A / \mathrm{B})$ plays an important role in the regulation of the bioconversion of acid precursors into solvents. The presence of the CtfA/B enzyme is consistently linked to solventogenesis 
(acetone and butanol), since it was observed that its downregulation lowers acetonebutanol production [116,117].

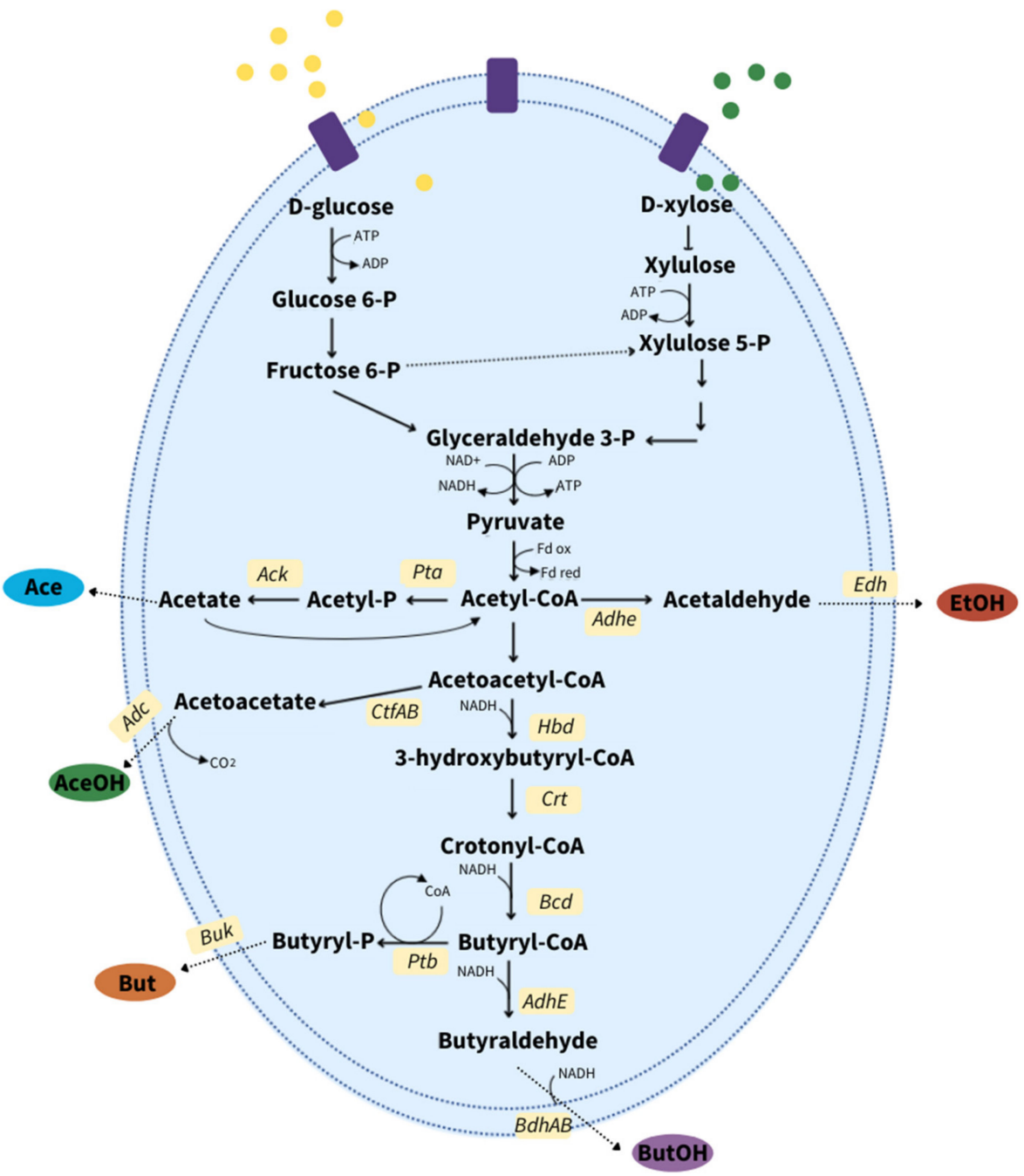

Figure 3. General metabolic pathway studied for value-added products on acidogenic and solventogenic phase performance. Ack: acetate kinase; Pta: phosphotransacetylase; Edh: ethanol dehydrogenase; Hbd: 3-hydroxybutyryl-CoA dehydrogenase; CtfAb: CoA transferase; Adc: acetoacetate decarboxylase; Crt: crotonase; Bcd: butyryl-CoA dehydrogenase; Ptb: phosphotransbutyrylase; Buk: butyrate kinase; AdhE: butyraldehyde dehydrogenase; BdhAB: butanol dehydrogenase; Ace: acetate; AceOH: acetone; EtOH: ethanol; But: butyrate; ButOH: butanol.

The metabolism switching from acid to solvent production has been analyzed as a strategy developed by microorganisms to alternate the intracellular stress. Despite its importance, solventogenesis still lacks fundamental understanding. Identification of mechanisms that regulate this process is important to increase production without bacterial population decay [118].

Furthermore, solvent butanol-acetone yield is well known to be coupled to $\mathrm{pH}$ decrease. At this point, the activity of CoA transferase (CtfAB) and aldehyde/alcohol dehydrogenase (AdhE1) increases, which induces the solventogenesis and transfer of electrons through flavodoxins, ferredoxin and thioredoxin [119]. The $\mathrm{H}_{2}$-uptake hydrogenases regulate the flow of electrons and are actively expressed during acidogenesis. Additionally, Nakayama et al. [120] indicated that energy transfer has a key role in solventogenesis and reported that hydrogenases were upregulated to increase acetone/butanol yield.

In another study, alcohol dehydrogenase (ADH) production was observed in Acinetobacter strains during ethanol conversion to acetate, the primary pathway of reversion 
of solventogenesis. The ADH has been related to bacterial quorum sensing, and as a key stimulator for alcohol oxidation [121]. Conversely, spore formation has been reported as a survival mechanism under solventogenesis stress. Spo0A has been identified as a global regulator of solvent production. The overexpression of this gene, spo0A, in C. acetobutylicum resulted in the upregulation of acetoacetate decarboxylase (CAP0165) and butanol dehydrogenase (CAC3299), while acetate kinase (CAC1743) and butyrate kinase (CAC2075/CAC1660) were downregulated [122].

\subsection{Proteomics of Methane and Hydrogen Production}

Production of biohydrogen and methane is undertaken by specific groups of microorganisms. Microorganisms from the genera Halothermothrix, Syntrophomonas and Clostridium are important players in the production of hydrogen [123]. Further, acetate accumulated during the processes is oxidized by syntrophic bacteria into $\mathrm{H}_{2}$ and $\mathrm{CO}_{2}$. Methanogenesis is a slow reaction and sensitive to inhibitory factors (e.g., ammonium and sulfide) [124]. Many studies have identified several enzymes associated with aceticlastic and hydrogenotrophic-methanogenesis pathway, viz., $\mathrm{F}_{420}$ non-reducing hydrogenase/heterodisulfide reductase complex, methylcoenzymeM reductase, tetrahydromethanopterin S-methyltransferase $[123,125]$. High abundance of enzymes involved in methanogenesis, either the key enzyme of hydrogenotrophs (5,10-methylenetetrahydromethanopterin reductase) or the acetoclastic methanogens (acetylCoA decarbonylase/synthase) are essential [126,127]. The most important step involved is the production and activity of the key enzyme, methyl coenzyme M reductase (encode by the gene $m c r A$ ) to increase methane production [128].

Methane production and organic acids production are reported to be directly correlated to methane percentage and molar values of acids (i.e., acetate) [129]. This fermentation step is correlated to all the further steps of production. NiFe and Fe-Fe hydrogenases are the most common and both use the $\mathrm{NAD}(\mathrm{P}) \mathrm{H}$ as a donor and reduce ferredoxin proteins for hydrogen production [130]. Further, periplasmatically oriented hydrogen-oxidizing and a cytoplasmatically oriented putative $\mathrm{H}_{2}$-producing membrane bound hydrogenases have been reported in Sulfospirillum multivorans [131].

The distinct protein production profile during biohydrogen and methanation is influenced by several factors that induce stress to the cell; for example, acid tolerance is one of the limitations that these processes face. A study reported that at $7.5 \mathrm{~g} / \mathrm{L}$ of butyrate presents a positive stress for protein abundance to overcome the stress and avoid affecting production. Nonetheless, when the concentration raised to $15 \mathrm{~g} / \mathrm{L}$, negative stress was observed lowering protein production. Performance of the process in the same study, reported the proton transfer as the main factor under this stress conditions of acidification, were dehydrogenases played a key role. The principal enzymes synthesized under acidification were dehydrogenase and methyltranferases proteins related to methane production [132]. Conversely, for sole $\mathrm{H}_{2}$ production the electron-transfer flavoprotein, hydrogenase expression/formation protein (hupG) and phosphate butyryl transferase (sp2) were known for their role in $\mathrm{H}_{2}$ production, especially the $\mathrm{sp} 2$ is mostly expressed when higher concentrations of butyrate are present, similarly to methane production [133].

Other studies report the influence of temperature on regulation and protein folding, where a decrease in temperature results in the overproduction of heat shock proteins (HSp). In biomethanation, it has been shown that the mechanisms of cells will change the regulation and high expression of genes encoding for nucleic-acid-binding proteins (CspA-related proteins) and chaperones (DnaK and GroEL) [134]. In contrast, at higher temperatures $\left(30-55{ }^{\circ} \mathrm{C}\right), \mathrm{Hsp} 70$ and Hsp60 enzyme stress systems are upregulated in order to assist protein folding. Under thermophilic conditions, HSp are thermostability indicators, although it has been found that protein synthesis levels of key enzymes involved in methane metabolism at high temperatures takes place. Hydrogenotrophic enzymes such as acetate kinase and the acetyl-CoA decarbonylase/synthase complex were detected at $55^{\circ} \mathrm{C}[135,136]$. Moreover, HSp are also present when other forms of stress affect the microbial community, such as high ammonium and high salt concentrations [135]. 


\section{Conclusions}

Although lignocellulose is a highly recalcitrant material, its abundance makes it an ideal candidate to produce biofuels, such as bioethanol and methane, as well as other products of value, such as organic solvents and acids. Hydrolysis of plant biomass components under anaerobic conditions is carried out by microbial consortia and several enzymes need to act synergistically. Members of Firmicutes, Bacteroidetes, Proteobacteria play a major role, where Clostridium spp. are the most well-studied cellulolytic bacteria and are also industrially exploited for cellulose catabolism. In general, cellulases and hemicellulases, which are GHs, hydrolyze glycosidic bonds. These GH enzymes are classified into more than one hundred families depending on their protein sequence. Besides $\mathrm{GH}$, other enzymes take part in the breakdown of cellulose including polysaccharide lyases, carbohydrate esterases and auxiliary activities (Table 1). Moreover, the production of methane requires the presence of multiple enzymes, primarily methyl coenzyme $\mathrm{M}$ reductase, whose upregulation is associated with increased methane production.

It is well known that the bioconversion of plant biomass requires several pretreatment strategies, including chemical, mechanical and enzymatic treatments or a combination of different methods (Table 4). Chemical pretreatments have been found to yield higher ethanol titers; however, chemical treatments result in the formation of several compounds that inhibit enzymatic activity later in the process. Additionally, several intermediates such as volatile fatty acids and alcohols formed may induce cellular toxicity. Thereby, making the process of biofuel production from plant biomass practical and efficient requires a thorough knowledge of the proteins, primarily the enzymes involved in the conversion of complex sugars into ethanol, methane and other compounds, and of the proteins involved in mechanisms of stress tolerance. In this context, proteomics is a promising technology that can be used to identify proteins of interest that could aid in identification and development of engineered microbial pathways and monitoring strategies. Hence, this review highlights the involvement of the wide variety of enzymatic proteins during the bioprocess of biofuel production from lignocelluosic feedstocks under anaerobic conditions.

Proteomics has been used earlier to characterize the proteins present in plant biomass, and these studies have primarily addressed the composition of plants cell walls from different sources $[137,138]$. Meanwhile, studies on the microbial proteome tend to focus on one microorganism growing in the presence of a particular polysaccharide. Earlier studies have shown the differences between important microbial enzymes when distinct microorganisms were grown in the presence of varied substrates (Table 5). Although several proteins involved in central and pyruvate metabolism as well as ethanol production have shown differential abundance, patterns among the substrates, conditions and microorganisms involved are imperceptible. Multivariable studies where the same complex inoculum or several bacteria are employed under diverse conditions, may reveal whether certain functional groups of proteins display similar production patterns. However, due to the high complexity behind biofuel production, it is our view that multivariable analyses may not reveal comprehensive principles that could be applied to all bioprocesses. Instead, we postulate that employing proteomic studies will aid to improve the design and application of a specific bioprocess, where distinct proteins could be identified as efficiency biomarkers at every stage of that unique process, or where certain enzymes could be targeted for metabolic engineering to increase production of a desired compound.

In general, previous studies have shown how proteomics could be applied in the context of biofuel production and for process improvement. For instance, quantitative proteomics by tandem mass spectrometry identified unique upregulated proteins corresponding to photosystems of a cyanobacterial species [139], which suggested that these proteins could be targets to design ethanol-tolerant superior strains. Similarly, proteomics analyses of a Clostridium strain grown on different carbon sources, including cellulose and hemicellulose, allowed the identification of key enzymes that participate in the breakdown of each distinct substrate, these enzymes could in turn be used as targets to engineer this bacterium to favor the uptake of a particular substrate [140]. In a more recent study using 
Clostridium cellulovorans, it was observed that global proteome profiles were carbon sourcedependent, with notable differences in the upregulation of ATP-biosynthesis enzymes in cells grown on cellulose [22]. Meanwhile, a study of Clostridum acetobutylicum grown on different polysaccharides, including lignin, identified multiple metabolic pathways and proteins that are repressed in the presence of lignin. These proteins included ATPdependent cell division factors, which were deemed part of the "lignin bottleneck" for this organism, and could be used as biomarkers to monitor the presence of this Clostridum strain or for modification and engineering [104] Moreover, another study focused on two cellulolytic Bacteroidetes strains using label-free protein quantification coupled with cell fractionation revealed proteins present when the strains grew on two distinct polysaccharides and their subcellular localization. The results identified strain-specific enzymes and previously unstudied GHs [141], and these proteins could also be used as biomarkers for these strains.

Thus, proteomics analyses can retrieve direct protein production signatures, such as accumulation or decrease of particular enzymes. These data could help in the identification of target enzymes that could be in turn engineered to avoid metabolic bottlenecks that are encountered in using lignocellulosic feedstocks. Additionally, proteins interact allosterically with multiple molecules, and hence proteomics studies coupled with crosslinking and massspectrometry-based identification in the context of bioconversion could be used to map such interactions, and in turn aid in the design of improved microbial strains engineered for optimal activity. At the moment, applications of proteomics are process-specific, but in the future, a combination of multiple biological scales, i.e., proteomics, transcriptomics and metabolomics, may lead to the development of machine learning tools that can predict and design strategies for the bioconversion of recalcitrant feedstocks into biofuels and other value-added products.

Author Contributions: Conceptualization, N.B. and A.A.; methodology, N.B. and A.A.; investigation, M.I.V.-M., A.G.T.-C., K.M.E.-U., A.A. and N.B.; validation, M.I.V.-M., S.S.-M., A.A. and N.B.; formal analysis, M.I.V.-M., A.G.T.-C., and S.S.-M.; resources, N.B.; data curation, M.I.V.-M. and S.S.-M.; writing—original draft preparation, M.I.V.-M., A.G.T.-C. and K.M.E.-U.; writing—review and editing, M.P.L.-E., F.H.-T., A.A. and N.B.; supervision, A.A. and N.B.; project administration, N.B.; funding acquisition, N.B. All authors have read and agreed to the published version of the manuscript.

Funding: This research was funded by Universidad Autónoma de Coahuila under the auspices of proyectos semillas DIP-UADEC C01-2021-105.

Data Availability Statement: Not applicable.

Acknowledgments: M.I.V.-M., A.G.T.-C. and K.M.E.-U acknowledge the scholarship received during their degree program.

Conflicts of Interest: The authors declare that their work was conducted in the absence of any commercial or financial relationships that could be interpreted as a potential conflict of interest.

\section{References}

1. Jiang, Y.; Xin, F.; Lu, J.; Dong, W.; Zhang, W.; Zhang, M.; Wu, H.; Ma, J.; Jiang, M. State of the art review of biofuels production from lignocellulose by thermophilic bacteria. Bioresour. Technol. 2017, 245, 1498-1506. [CrossRef] [PubMed]

2. Oh, Y.K.; Hwang, K.R.; Kim, C.; Kim, J.R.; Lee, J.S. Recent developments and key barriers to advanced biofuels: A short review. Bioresour. Technol. 2018, 257, 320-333. [CrossRef] [PubMed]

3. Rosnow, J.J.; Anderson, L.N.; Nair, R.N.; Baker, E.S.; Wright, A.T. Profiling microbial lignocellulose degradation and utilization by emergent omics technologies. Crit. Rev. Biotechnol. 2016, 37, 626-640. [CrossRef] [PubMed]

4. Wi, S.G.; Cho, E.J.; Lee, D.-S.; Lee, S.J.; Lee, Y.J.; Bae, H.-J. Lignocellulose conversion for biofuel: A new pretreatment greatly improves downstream biocatalytic hydrolysis of various lignocellulosic materials. Biotechnol. Biofuels 2015, 8, 1-11. [CrossRef]

5. Shen, L.; Su, Y.; Sun, Y.; Wang, G.; Chen, H.; Yu, X.; Zhang, S.; Chen, G. Establishment of a highly efficient and low cost mixed cellulase system for bioconversion of corn stover by Trichoderma reesei and Aspergillus niger. Biocatal. Agric. Biotechnol. 2021, 32, 101849. [CrossRef]

6. Lee, J. Biological conversion of lignocellulosic biomass to ethanol. J. Biotechnol. 1997, 56, 1-24. [CrossRef]

7. Liu, Y.; Li, B.; Feng, Y.; Cui, Q. Consolidated bio-saccharification: Leading lignocellulose bioconversion into the real world. Biotechnol. Adv. 2020, 40, 107535. [CrossRef] [PubMed] 
8. Akinosho, H.; Yee, K.; Close, D.; Ragauskas, A. The emergence of Clostridium thermocellum as a high utility candidate for consolidated bioprocessing applications. Front. Chem. 2014, 2, 66. [CrossRef] [PubMed]

9. Shinoda, S.; Kurosaki, M.; Kokuzawa, T.; Hirano, K.; Takano, H.; Ueda, K.; Hirano, N. Comparative Biochemical Analysis of Cellulosomes Isolated from Clostridium clariflavum DSM 19732 and Clostridium thermocellum ATCC 27405 Grown on Plant Biomass. Appl. Biochem. Biotechnol. 2018, 187, 994-1010. [CrossRef] [PubMed]

10. Qi, K.; Chen, C.; Yan, F.; Feng, Y.; Bayer, E.A.; Kosugi, A.; Liu, Y.-J. Coordinated $\beta$-glucosidase activity with the cellulosome is effective for enhanced lignocellulose saccharification. Bioresour. Technol. 2021, 337, 125441. [CrossRef]

11. Basak, B.; Ahn, Y.; Kumar, R.; Hwang, J.; Kim, K.; Jeon, B. Lignocellulolytic microbiomes for augmenting lignocellulose degradation in anaerobic digestion. Trends Microbiol. 2021. [CrossRef] [PubMed]

12. Antunes, F.A.F.; Chandel, A.K.; Terán-Hilares, R.; Ingle, A.P.; Rai, M.; dos Santos Milessi, T.S.; da Silva, S.S.; Dos Santos, J.C. Overcoming challenges in lignocellulosic biomass pretreatment for second-generation $(2 \mathrm{G})$ sugar production: Emerging role of nano, biotechnological and promising approaches. 3 Biotech. 2019, 9, 1-17. [CrossRef] [PubMed]

13. Olajuyigbe, F.M.; Fatokun, C.O.; Oyelere, O.M. Biodelignification of some agro-residues by Stenotrophomonas sp. CFB-09 and enhanced production of ligninolytic enzymes. Biocatal. Agric. Biotechnol. 2018, 15, 120-130. [CrossRef]

14. Tsegaye, B.; Balomajumder, C.; Roy, P. Biodelignification and hydrolysis of rice straw by novel bacteria isolated from wood feeding termite. 3 Biotech. 2018, 8, 1-11. [CrossRef]

15. Hidayatullah, I.M.; Al Husna, M.D.; Radiyan, H.; Kresnowati, M.T.A.P.; Suhardi, S.H.; Setiadi, T.; Boopathy, R. Combining biodelignification and hydrothermal pretreatment of oil palm empty fruit bunches (OPEFB) for monomeric sugar production. Bioresour. Technol. Rep. 2021, 15, 100808. [CrossRef]

16. Li, X.; Wu, X.; Yue, W.; Liu, J.; Li, G.L.; Miao, Y. Proteomic Analysis of the Silkworm (Bombyx mori L.) Hemolymph during Developmental Stage. J. Proteome Res. 2006, 5, 2809-2814. [CrossRef] [PubMed]

17. Aslam, B.; Basit, M.; Nisar, M.A.; Khurshid, M.; Rasool, M.H. Proteomics: Technologies and Their Applications. J. Chromatogr. Sci. 2016, 55, 182-196. [CrossRef]

18. Twyman, R.M. Proteomics. Encycl. Appl. Ethics 2012, 642-649. [CrossRef]

19. Cunha, B.; Aguiar, T.; Carvalho, S.; Silva, M.; Gomes, R.; Carrondo, M.; Gomes-Alves, P.; Peixoto, C.; Serra, M.; Alves, P.M. Bioprocess integration for human mesenchymal stem cells: From up to downstream processing scale-up to cell proteome characterization. J. Biotechnol. 2017, 248, 87-98. [CrossRef] [PubMed]

20. Zheng, Y.; Chang, Y.; Xie, S.; Song, J.; Wang, M. Impacts of bioprocess engineering on product formation by Acetobacter pasteurianus. Appl. Microbiol. Biotechnol. 2018, 102, 2535-2541. [CrossRef] [PubMed]

21. Ali, A.S.; Raju, R.; Kshirsagar, R.; Ivanov, A.R.; Gilbert, A.; Zang, L.; Karger, B.L. Multi-Omics Study on the Impact of Cysteine Feed Level on Cell Viability and mAb Production in a CHO Bioprocess. Biotechnol. J. 2018, 14, 1800352. [CrossRef]

22. Usai, G.; Cirrincione, S.; Re, A.; Manfredi, M.; Pagnani, A.; Pessione, E.; Mazzoli, R. Clostridium cellulovorans metabolism of cellulose as studied by comparative proteomic approach. J. Proteom. 2020, 216, 103667. [CrossRef]

23. Saykhedkar, S.; Ray, A.; Ayoubi-Canaan, P.; Hartson, S.; Prade, R.; Mort, A. A time course analysis of the extracellular proteome of Aspergillus nidulans growing on sorghum stover. Biotechnol. Biofuels 2012, 5, 52. [CrossRef] [PubMed]

24. Tian, X.; Chen, L.; Wang, J.; Qiao, J.; Zhang, W. Quantitative proteomics reveals dynamic responses of Synechocystis sp. PCC 6803 to next-generation biofuel butanol. J. Proteom. 2013, 78, 326-345. [CrossRef] [PubMed]

25. Liu, J.; Chen, L.; Wang, J.; Qiao, J.; Zhang, W. Proteomic analysis reveals resistance mechanism against biofuel hexane in Synechocystis sp. PCC 6803. Biotechnol. Biofuels 2012, 5, 68. [CrossRef]

26. Jain, S.; Graham, C.; Graham, R.; McMullan, G.; Ternan, N. Quantitative Proteomic Analysis of the Heat Stress Response in Clostridium difficile Strain 630. J. Proteome Res. 2011, 10, 3880-3890. [CrossRef] [PubMed]

27. Farrell, A.; McLoughlin, N.; Milne, J.J.; Marison, I.W.; Bones, J. Application of Multi-Omics Techniques for Bioprocess Design and Optimization in Chinese Hamster Ovary Cells. J. Proteome Res. 2014, 13, 3144-3159. [CrossRef] [PubMed]

28. Campbell, K.; Xia, J.; Nielsen, J. The Impact of Systems Biology on Bioprocessing. Trends Biotechnol. 2017, 35, 1156-1168. [CrossRef] [PubMed]

29. Baycin-Hizal, D.; Tabb, D.L.; Chaerkady, R.; Chen, L.; Lewis, N.E.; Nagarajan, H.; Betenbaugh, M. Proteomic Analysis of Chinese Hamster Ovary Cells. J. Proteome Res. 2012, 11, 5265-5276. [CrossRef] [PubMed]

30. Andlar, M.; Rezić, T.; Marđetko, N.; Kracher, D.; Ludwig, R.; Šantek, B. Lignocellulose degradation: An overview of fungi and fungal enzymes involved in lignocellulose degradation. Eng. Life Sci. 2018, 18, 768-778. [CrossRef] [PubMed]

31. Li, H.; Wu, H.; Xiong, L.; Chen, X.; Wang, C.; Qi, G.; Chen, X. The hydrolytic efficiency and synergistic action of recombinant xylan-degrading enzymes on xylan isolated from sugarcane bagasse. Carbohydr. Polym. 2017, 175, 199-206. [CrossRef] [PubMed]

32. Cagide, C.; Castro-Sowinski, S. Technological and biochemical features of lignin-degrading enzymes: A brief review. Environ. Sustain. 2020, 3, 371-389. [CrossRef]

33. Binod, P.; Gnansounou, E.; Sindhu, R.; Pandey, A. Enzymes for second generation biofuels: Recent developments and future perspectives. Bioresour. Technol. Rep. 2019, 5, 317-325. [CrossRef]

34. Conway, J.M.; Crosby, J.R.; Hren, A.P.; Southerland, R.T.; Lee, L.L.; Lunin, V.V.; Alahuhta, P.; Himmel, M.E.; Bomble, Y.; Adams, M.W.W.; et al. Novel multidomain, multifunctional glycoside hydrolases from highly lignocellulolytic Caldicellulosiruptor species. AIChE J. 2018, 64, 4218-4228. [CrossRef] 
35. Babar, M.M.; Afzaal, H.; Pothineni, V.R.; Zaidi, N.-S.S.; Ali, Z.; Zahid, M.A.; Gul, A. Omics Approaches in Industrial Biotechnology and Bioprocess Engineering. In Omics Technologies and Bio-Engineering; Academic Press: Cambridge, MA, USA, 2018; pp. 251-269. [CrossRef]

36. Zhang, Z.; Wu, S.; Stenoien, D.L.; Paša-Tolić, L. High-Throughput Proteomics. Annu. Rev. Anal. Chem. 2014, 7, 427-454. [CrossRef] [PubMed]

37. Heffner, K.; Hizal, D.; Kumar, A.; Shiloach, J.; Zhu, J.; Bowen, M.; Betenbaugh, M. Exploiting the proteomics revolution in biotechnology: From disease and antibody targets to optimizing bioprocess development. Curr. Opin. Biotechnol. 2014, 30, 80-86. [CrossRef] [PubMed]

38. Sharma, H.K.; Xu, C.; Qin, W. Biological Pretreatment of Lignocellulosic Biomass for Biofuels and Bioproducts: An Overview. Waste Biomass Valorization 2017, 10, 235-251. [CrossRef]

39. Pellegrini, V.; Sepulchro, A.; Polikarpov, I. Enzymes for lignocellulosic biomass polysaccharide valorization and production of nanomaterials. Curr. Opin. Green Sustain. Chem. 2020, 26, 100397. [CrossRef]

40. Pu, Y.; Hu, F.; Huang, F.; Davison, B.H.; Ragauskas, A.J. Assessing the molecular structure basis for biomass recalcitrance during dilute acid and hydrothermal pretreatments. Biotechnol. Biofuels 2013, 6, 15. [CrossRef] [PubMed]

41. Himmel, M.E.; Ding, S.-Y.; Johnson, D.K.; Adney, W.S.; Nimlos, M.R.; Brady, J.W.; Foust, T.D. Biomass Recalcitrance: Engineering Plants and Enzymes for Biofuels Production. Science 2007, 315, 804-807. [CrossRef] [PubMed]

42. Guo, H.; Wang, X.-D.; Lee, D.-J. Proteomic researches for lignocellulose-degrading enzymes: A mini-review. Bioresour. Technol. 2018, 265, 532-541. [CrossRef] [PubMed]

43. Saha, B.C. Hemicellulose bioconversion. J. Ind. Microbiol. Biotechnol. 2003, 30, 279-291. [CrossRef] [PubMed]

44. Kulasinski, K.; Keten, S.; Churakov, S.V.; Derome, D.; Carmeliet, J. A comparative molecular dynamics study of crystalline, paracrystalline and amorphous states of cellulose. Cellulose 2014, 21, 1103-1116. [CrossRef]

45. Li, X.; Griffin, K.; Langeveld, S.; Frommhagen, M.; Underlin, E.N.; Kabel, M.A.; de Vries, R.P.; Dilokpimol, A. Functional Validation of Two Fungal Subfamilies in Carbohydrate Esterase Family 1 by Biochemical Characterization of Esterases From Uncharacterized Branches. Front. Bioeng. Biotechnol. 2020, 8, 694. [CrossRef] [PubMed]

46. Lombard, V.; Golaconda Ramulu, H.; Drula, E.; Coutinho, P.M.; Henrissat, B. The carbohydrate-active enzymes database (CAZy) in 2013. Nucleic Acids Res. 2013, 42, 490-495. [CrossRef] [PubMed]

47. Yang, T.; Li, X.; Guo, Y.; Peng, S.; Liu, G.; Zhao, J. Effect of endoglucanases from different glycoside hydrolase families on enzymatic preparation of cellulose nanocrystal. Ind. Crop. Prod. 2020, 155, 112755. [CrossRef]

48. Chuzel, L.; Ganatra, M.B.; Rapp, E.; Henrissat, B.; Taron, C.H. Functional metagenomics identifies an exosialidase with an inverting catalytic mechanism that defines a new glycoside hydrolase family (GH156). J. Biol. Chem. 2018, 293, 18138-18150. [CrossRef] [PubMed]

49. Okuyama, M.; Kitamura, M.; Hondoh, H.; Kang, M.-S.; Mori, H.; Kimura, A.; Yao, M. Catalytic Mechanism of Retaining $\alpha$-Galactosidase Belonging to Glycoside Hydrolase Family 97. J. Mol. Biol. 2009, 392, 1232-1241. [CrossRef] [PubMed]

50. Thygesen, A.; Oddershede, J.; Lilholt, H.; Thomsen, A.B.; Ståhl, K. On the determination of crystallinity and cellulose content in plant fibres. Cellulose 2005, 12, 563-576. [CrossRef]

51. Turner, B.L.; Hopkins, D.W.; Haygarth, P.M.; Ostle, N. $\beta$-Glucosidase activity in pasture soils. Appl. Soil Ecol. 2002, 20, 157-162. [CrossRef]

52. Zhu, N.; Yang, J.; Ji, L.; Liu, J.; Yang, Y.; Yuan, H. Metagenomic and metaproteomic analyses of a corn stover-adapted microbial consortium EMSD5 reveal its taxonomic and enzymatic basis for degrading lignocellulose. Biotechnol. Biofuels 2016, 9, 1-23. [CrossRef] [PubMed]

53. Munir, R.I.; Spicer, V.; Shamshurin, D.; Krokhin, O.V.; Wilkins, J.; Ramachandran, U.; Sparling, R.; Levin, B.D. Quantitative proteomic analysis of the cellulolytic system of Clostridium termitidis CT1112 reveals distinct protein expression profiles upon growth on $\alpha$-cellulose and cellobiose. J. Proteom. 2015, 125, 41-53. [CrossRef] [PubMed]

54. Ma, J.; Zhang, K.; Liao, H.; Hector, S.B.; Shi, X.; Li, J.; Liu, B.; Xu, T.; Tong, C.; Liu, X.; et al. Genomic and secretomic insight into lignocellulolytic system of an endophytic bacterium Pantoea ananatis Sd-1. Biotechnol. Biofuels 2016, 9, 1-15. [CrossRef] [PubMed]

55. Wongwilaiwalin, S.; Rattanachomsri, U.; Laothanachareon, T.; Eurwilaichitr, L.; Igarashi, Y.; Champreda, V. Analysis of a thermophilic lignocellulose degrading microbial consortium and multi-species lignocellulolytic enzyme system. Enzym. Microb. Technol. 2010, 47, 283-290. [CrossRef]

56. Lochner, A.; Giannone, R.J.; Rodriguez, M., Jr.; Shah, M.B.; Mielenz, J.R.; Keller, M.; Graham, D.E.; Hettich, R.L. Use of Label-Free Quantitative Proteomics To Distinguish the Secreted Cellulolytic Systems of Caldicellulosiruptor bescii and Caldicellulosiruptor obsidiansis. Appl. Environ. Microbiol. 2011, 77, 4042-4054. [CrossRef] [PubMed]

57. Gold, N.D.; Martin, V.J.J. Global View of the Clostridium thermocellum Cellulosome Revealed by Quantitative Proteomic Analysis. J. Bacteriol. 2007, 189, 6787-6795. [CrossRef] [PubMed]

58. Morisaka, H.; Matsui, K.; Tatsukami, Y.; Kuroda, K.; Miyake, H.; Tamaru, Y.; Ueda, M. Profile of native cellulosomal proteins of Clostridium cellulovorans adapted to various carbon sources. AMB Expr. 2012, 2, 37. [CrossRef]

59. Blouzard, J.-C.; Coutinho, P.M.; Fierobe, H.-P.; Henrissat, B.; Lignon, S.; Tardif, C.; de Philip, P. Modulation of cellulosome composition in Clostridium cellulolyticum: Adaptation to the polysaccharide environment revealed by proteomic and carbohydrateactive enzyme analyses. Proteomics 2010, 10, 541-554. [CrossRef] 
60. Kumar, M.; Verma, S.; Gazara, R.K.; Kumar, M.; Pandey, A.; Verma, P.K.; Thakur, I.S. Genomic and proteomic analysis of lignin degrading and polyhydroxyalkanoate accumulating $\beta$-proteobacterium Pandoraea sp. ISTKB. Biotechnol. Biofuels 2018, 11, 154. [CrossRef] [PubMed]

61. Li, J.; Du, X.; Feng, Q.; Yan, H. Extracellular differential proteome analysis of substrates of different lignin model compounds degraded by Aspergillus fumigatus G-13. J. Environ. Eng. Landsc. Manag. 2020, 28, 137-147. [CrossRef]

62. Ravalason, H.; Jan, G.; Mollé, D.; Pasco, M.; Coutinho, P.M.; Lapierre, C.; Pollet, B.; Bertaud, F.; Petit-Conil, M.; Grisel, S.; et al. Secretome analysis of Phanerochaete chrysosporium strain CIRM-BRFM41 grown on softwood. Appl. Microbiol. Biotechnol. 2008, 80, 719. [CrossRef]

63. Shallom, D.; Shoham, Y. Microbial hemicellulases. Curr. Opin. Microbiol. 2003, 6, 219-228. [CrossRef]

64. Nakamura, A.; Nascimento, A.; Polikarpov, I. Structural diversity of carbohydrate esterases. Biotechnol. Res. Innov. 2017, 1, 35-51. [CrossRef]

65. Mäkelä, M.; Dilokpimol, A.; Koskela, S.; Kuuskeri, J.; de Vries, R.; Hildén, K. Characterization of a feruloyl esterase from Aspergillus terreus facilitates the division of fungal enzymes from Carbohydrate Esterase family 1 of the carbohydrate-active enzymes (CAZy) database. Microb. Biotechnol. 2018, 11, 869-880. [CrossRef] [PubMed]

66. Chauhan, P. Role of various bacterial enzymes in complete depolymerization of lignin: A review. Biocatal. Agric. Biotechnol. 2020, 23, 101498. [CrossRef]

67. Levasseur, A.; Drula, E.; Lombard, V.; Coutinho, P.M.; Henrissat, B. Expansion of the enzymatic repertoire of the CAZy database to integrate auxiliary redox enzymes. Biotechnol. Biofuels 2013, 6, 41. [CrossRef] [PubMed]

68. Reiter, J.; Strittmatter, H.; Wiemann, L.O.; Schieder, D.; Sieber, V. Enzymatic cleavage of lignin $\beta$-O-4 aryl ether bonds via net internal hydrogen transfer. Green Chem. 2013, 15, 1373. [CrossRef]

69. Picart, P.; de María, P.D.; Schallmey, A. From gene to biorefinery: Microbial $\beta$-etherases as promising biocatalysts for lignin valorization. Front. Microbiol. 2015, 6, 916. [CrossRef] [PubMed]

70. Araki, T.; Tanatani, K.; Kamimura, N.; Otsuka, Y.; Yamaguchi, M.; Nakamura, M.; Masai, E. Sphingobium sp. SYK-6 syringate O-demethylase gene is regulated by DesX, unlike other vanillate and syringate catabolic genes regulated by DesR. Appl. Environ. Microbiol. 2020, 86, e01712-20. [CrossRef] [PubMed]

71. Gall, D.L.; Ralph, J.; Donohue, T.J.; Noguera, D.R. Benzoyl Coenzyme A Pathway-Mediated Metabolism of meta-HydroxyAromatic Acids in Rhodopseudomonas palustris. J. Bacteriol. 2013, 195, 4112-4120. [CrossRef] [PubMed]

72. Philipp, B.; Kemmler, D.; Hellstern, J.; Gorny, N.; Caballero, A.; Schink, B. Anaerobic degradation of protocatechuate (3,4dihydroxybenzoate) by Thauera aromaticastrain AR-1. FEMS Microbiol. Lett. 2002, 212, 139-143. [CrossRef]

73. Boll, M. Key enzymes in the anaerobic aromatic metabolism catalysing Birch-like reductions. Biochim. Biophys. Acta (BBA) Bioenerg. 2005, 1707, 34-50. [CrossRef]

74. Wischgoll, S.; Heintz, D.; Peters, F.; Erxleben, A.; Sarnighausen, E.; Reski, R.; Dorsselaer, A.V.; Boll, M. Gene clusters involved in anaerobic benzoate degradation of Geobacter metallireducens. Mol. Microbiol. 2005, 58, 1238-1252. [CrossRef]

75. Carmona, M.; Zamarro, M.T.; Blazquez, B.; Durante-Rodriguez, G.; Juarez, J.F.; Valderrama, J.A.; Barragan, M.J.L.; Garcia, J.L.; Diaz, E. Anaerobic Catabolism of Aromatic Compounds: A Genetic and Genomic View. Microbiol. Mol. Biol. Rev. 2009, 73, 71-133. [CrossRef] [PubMed]

76. Harwood, C.S.; Parales, R.E. tThe $\beta$-ketoadipate pathway and the biology of self-identity. Annu. Rev. Microbiol. 1996, 50, 553-590. [CrossRef] [PubMed]

77. JimenezJiménez, N.; Curiel, J.A.; Reveron, I.; de las Rivas, B.; Munoz, R. Uncovering the Lactobacillus plantarum WCFS1 Gallate Decarboxylase Involved in Tannin Degradation. Appl. Environ. Microbiol. 2013, 79, 4253-4263. [CrossRef] [PubMed]

78. Reichenbecher, W.; Schink, B. Towards the reaction mechanism of pyrogallol-phloroglucinol transhydroxylase of Pelobacter acidigallici. Biochim. Biophys. Acta (BBA) Protein Struct. Mol. Enzymol. 1999, 1430, 245-253. [CrossRef]

79. Brune, A.; Schink, B. Phloroglucinol pathway in the strictly anaerobic Pelobacter acidigallici: Fermentation of trihydroxybenzenes to acetate via triacetic acid. Arch. Microbiol. 1992, 157, 417-424. [CrossRef]

80. Otsuka, Y.; Sonoki, T.; Ikeda, S.; Kajita, S.; Nakamura, M.; Katayama, Y. Detection and characterization of a novel extracellular fungal enzyme that catalyzes the specific and hydrolytic cleavage of lignin guaiacylglycerol beta-aryl ether linkages. Eur. J. Biochem. 2003, 270, 2353-2362. [CrossRef] [PubMed]

81. Husarcíková, J.; Voß, H.; de María, P.D.; Schallmey, A. Microbial $\beta$-etherases and glutathione lyases for lignin valorization in biorefineries: Current state and future perspectives. Appl. Microbiol. Biotechnol. 2018, 102, 5391-5401. [CrossRef] [PubMed]

82. Kato, S.; Chino, K.; Kamimura, N.; Masai, E.; Yumoto, I.; Kamagata, Y. Methanogenic degradation of lignin-derived monoaromatic compounds by microbial enrichments from rice paddy field soil. Sci. Rep. 2015, 5, 1-11. [CrossRef] [PubMed]

83. Porter, A.W.; Young, L.Y. Benzoyl-CoA, a Universal Biomarker for Anaerobic Degradation of Aromatic Compounds. Adv. Appl. Microbiol. 2014, 88, 167-203. [CrossRef]

84. Wells, T.; Ragauskas, A. Biotechnological opportunities with the $\beta$-ketoadipate pathway. Trends Biotechnol. 2012, 30, 627-637. [CrossRef]

85. Billings, A.F.; Fortney, J.L.; Hazen, T.C.; Simmons, B.; Davenport, K.W.; Goodwin, L.; Ivanova, N.; Mavromatis, K.; Woyke, T.; DeAngelis, K.M. Genome sequence and description of the anaerobic lignin-degrading bacterium Tolumonas lignolytica sp. nov. Stand. Genom. Sci. 2015, 10, 1-11. [CrossRef] [PubMed] 
86. Levy-Booth, D.J.; Hashimi, A.; Roccor, R.; Liu, L.Y.; Renneckar, S.; Eltis, W.; Mohn, W.W. Genomics and metatranscriptomics of biogeochemical cycling and degradation of lignin-derived aromatic compounds in thermal swamp sediment. ISME J. 2021, 15, 879-893. [CrossRef] [PubMed]

87. Stöcker, M. Biofuels and Biomass-To-Liquid Fuels in the Biorefinery: Catalytic Conversion of Lignocellulosic Biomass using Porous Materials. Angew. Chem. Int. Ed. 2008, 47, 9200-9211. [CrossRef] [PubMed]

88. Tsai, T.Y.; Lo, Y.C.; Dong, C.D.; Nagarajan, D.; Chang, J.S.; Lee, D.J. Biobutanol production from lignocellulosic biomass using immobilized Clostridium acetobutylicum. Appl. Energy 2020, 277, 115531. [CrossRef]

89. Wu, J.; Dong, L.; Liu, B.; Xing, D.; Zhou, C.; Wang, Q.; Wu, X.; Feng, L.; Cao, G. A novel integrated process to convert cellulose and hemicellulose in rice straw to biobutanol. Environ. Res. 2020, 186, 109580. [CrossRef] [PubMed]

90. Ranjan, A.; Khanna, S.; Moholkar, V.S. Feasibility of rice straw as alternate substrate for biobutanol production. Appl. Energy 2013, 103, 32-38. [CrossRef]

91. Ko, J.; Enkh-Amgalan, T.; Gong, G.; Um, Y.; Lee, S. Improved bioconversion of lignocellulosic biomass by Saccharomyces cerevisiae engineered for tolerance to acetic acid. GCB Bioenergy 2019, 12, 90-100. [CrossRef]

92. Menon, V.; Prakash, G.; Prabhune, A.; Rao, M. Biocatalytic approach for the utilization of hemicellulose for ethanol production from agricultural residue using thermostable xylanase and thermotolerant yeast. Bioresour. Technol. 2010, 101, 5366-5373. [CrossRef] [PubMed]

93. Reis, A.; Damilano, E.; Menezes, R.; de Morais, M., Jr. Second-generation ethanol from sugarcane and sweet sorghum bagasses using the yeast Dekkera bruxellensis. Ind. Crop. Prod. 2016, 92, 255-262. [CrossRef]

94. Huang, C.; Guo, H.J.; Wang, C.; Xiong, L.; Luo, M.T.; Chen, X.F.; Zhang, H.-R.; Li, H.-L.; Chen, X.-D. Efficient continuous biogas production using lignocellulosic hydrolysates as substrate: A semi-pilot scale long-term study. Energy Convers. Manag. 2017, 151, 53-62. [CrossRef]

95. Arreola-Vargas, J.; Ojeda-Castillo, V.; Snell-Castro, R.; Corona-González, R.; Alatriste-Mondragón, F.; Méndez-Acosta, H. Methane production from acid hydrolysates of Agave tequilana bagasse: Evaluation of hydrolysis conditions and methane yield. Bioresour. Technol. 2015, 181, 191-199. [CrossRef] [PubMed]

96. Cheng, X.; Liu, C. Enhanced coproduction of hydrogen and methane from cornstalks by a three-stage anaerobic fermentation process integrated with alkaline hydrolysis. Bioresour. Technol. 2012, 104, 373-379. [CrossRef] [PubMed]

97. Zhang, M.; Fan, Y.; Xing, Y.; Pan, C.; Zhang, G.; Lay, J. Enhanced biohydrogen production from cornstalk wastes with acidification pretreatment by mixed anaerobic cultures. Biomass Bioenergy 2007, 31, 250-254. [CrossRef]

98. Fan, Y.T.; Zhang, Y.H.; Zhang, S.F.; Hou, H.W.; Ren, B.Z. Efficient conversion of wheat straw wastes into biohydrogen gas by cow dung compost. Bioresour. Technol. 2006, 97, 500-505. [CrossRef] [PubMed]

99. Hernández-Beltrán, J.U.; Hernández-De Lira, I.O.; Cruz-Santos, M.M.; Saucedo-Luevanos, A.; Hernández-Terán, F.; Balagurusamy, N. Insight into Pretreatment Methods of Lignocellulosic Biomass to Increase Biogas Yield: Current State, Challenges, and Opportunities. Appl. Sci. 2019, 9, 3721. [CrossRef]

100. Bilal, M.; Iqbal, H.M.N.; Hu, H.; Wang, W.; Zhang, X. Metabolic engineering and enzyme-mediated processing: A biotechnological venture towards biofuel production-A review. Renew. Sustain. Energy Rev. 2018, 82, 436-447. [CrossRef]

101. Qian, E.W. Pretreatment and Saccharification of Lignocellulosic Biomass. Res. Approaches Sustain. Biomass Syst. 2014, 181-204. [CrossRef]

102. Li, H.; Mei, X.; Liu, B.; Xie, G.; Ren, N.; Xing, D. Quantitative proteomic analysis reveals the ethanologenic metabolism regulation of Ethanoligenens harbinense by exogenous ethanol addition. Biotechnol. Biofuels 2019, 12, 1-12. [CrossRef] [PubMed]

103. Li, H.; Mei, X.; Liu, B.; Li, Z.; Wang, B.; Ren, N.; Xing, D. Insights on acetate-ethanol fermentation by hydrogen-producing Ethanoligenens under acetic acid accumulation based on quantitative proteomics. Environ. Int. 2019, 129, 1-9. [CrossRef]

104. Raut, M.P.; Couto, N.; Pham, T.K.; Evans, C.; Noirel, J.; Wright, P.C. Quantitative proteomic analysis of the influence of lignin on biofuel production by Clostridium acetobutylicum ATCC 824. Biotechnol. Biofuels 2016, 9, 1-16. [CrossRef] [PubMed]

105. Poudel, S.; Giannone, R.J.; Basen, M.; Nookaew, I.; Poole, F.L.; Kelly, R.M.; Adams, M.W.W.; Hettich, R.L. The diversity and specificity of the extracellular proteome in the cellulolytic bacterium Caldicellulosiruptor bescii is driven by the nature of the cellulosic growth substrate. Biotechnol. Biofuels 2018, 11, 1-18. [CrossRef]

106. Traut, T. Phosphofructokinase. Allosteric Regul. Enzym. 2008, 139-159. [CrossRef]

107. Linton, K.J. Structure and Function of ABC Transporters. Physiology 2007, 22, 122-130. [CrossRef] [PubMed]

108. Zurawski, J.V.; Conway, J.M.; Lee, L.L.; Simpson, H.J.; Izquierdo, J.A.; Blumer-Schuette, S.; Nookaew, I.; Adams, M.W.W.; Kelly, R.M. Comparative Analysis of Extremely Thermophilic Caldicellulosiruptor Species Reveals Common and Unique Cellular Strategies for Plant Biomass Utilization. Appl. Environ. Microbiol. 2015, 81, 7159-7170. [CrossRef] [PubMed]

109. Van Lis, R.; Popek, M.; Couté, Y.; Kosta, A.; Drapier, D.; Nitschke, W.; Atteia, A. Concerted Up-regulation of Aldehyde/Alcohol Dehydrogenase (ADHE) and Starch in Chlamydomonas reinhardtii Increases Survival under Dark Anoxia. J. Biol. Chem. 2017, 292, 2395-2410. [CrossRef]

110. Dai, Z.; Dong, H.; Zhang, Y.; Li, Y. Elucidating the contributions of multiple aldehyde/alcohol dehydrogenases to butanol and ethanol production in Clostridium acetobutylicum. Sci. Rep. 2016, 6, 1-9. [CrossRef] [PubMed]

111. Lütke-Eversloh, T.; Bahl, H. Metabolic engineering of Clostridium acetobutylicum: Recent advances to improve butanol production. Curr. Opin. Biotechnol. 2011, 22, 634-647. [CrossRef] 
112. Groeger, C.; Wang, W.; Sabra, W.; Utesch, T.; Zeng, A.-P. Metabolic and proteomic analyses of product selectivity and redox regulation in Clostridium pasteurianum grown on glycerol under varied iron availability. Microb. Cell Factories 2017, 16, 1-16. [CrossRef] [PubMed]

113. Janssen, H.; Döring, C.; Ehrenreich, A.; Voigt, B.; Hecker, M.; Bahl, H.; Fischer, R.J. A proteomic and transcriptional view of acidogenic and solventogenic steady-state cells of Clostridium acetobutylicum in a chemostat culture. Appl. Microbiol. Biotechnol. 2010, 87, 2209-2226. [CrossRef] [PubMed]

114. Patakova, P.; Branska, B.; Sedlar, K.; Vasylkivska, M.; Jureckova, K.; Kolek, J.; Koscova, P.; Provaznik, I. Acidogenesis, solventogenesis, metabolic stress response and life cycle changes in Clostridium beijerinckii NRRL B-598 at the transcriptomic level. Sci. Rep. 2019, 9, 1-21. [CrossRef] [PubMed]

115. Li, S.; Huang, L.; Ke, C.; Pang, Z.; Liu, L. Pathway dissection, regulation, engineering and application: Lessons learned from biobutanol production by solventogenic clostridia. Biotechnol. Biofuels 2020, 13, 1-25. [CrossRef]

116. Haus, S.; Jabbari, S.; Millat, T.; Janssen, H.; Fischer, R.-J.; Bahl, H.; King, J.R.; Wolkenhauer, O. A systems biology approach to investigate the effect of $\mathrm{pH}$-induced gene regulation on solvent production by Clostridium acetobutylicum in continuous culture. BMC Syst. Biol. 2011, 5, 10. [CrossRef]

117. Liao, C.; Seo, S.O.; Lu, T. System-level modeling of acetone-butanol-ethanol fermentation. FEMS Microbiol. Lett. 2016, 363 , 74. [CrossRef]

118. Chakraborty, S.; Rene, E.R.; Lens, P.N.L.; Veiga, M.C.; Kennes, C. Enrichment of a solventogenic anaerobic sludge converting carbon monoxide and syngas into acids and alcohols. Bioresour. Technol. 2019, 272, 130-136. [CrossRef]

119. Jang, Y.S.; Han, M.J.; Lee, J.; Im, J.A.; Lee, Y.H.; Papoutsakis, E.T.; Bennett, G.; Lee, S.Y. Proteomic analyses of the phase transition from acidogenesis to solventogenesis using solventogenic and non-solventogenic Clostridium acetobutylicum strains. Appl. Microbiol. Biotechnol. 2014, 98, 5105-5115. [CrossRef]

120. Nakayama, S.; Kosaka, T.; Hirakawa, H.; Matsuura, K.; Yoshino, S.; Furukawa, K. Metabolic engineering for solvent productivity by downregulation of the hydrogenase gene cluster hupCBA in Clostridium saccharoperbutylacetonicum strain N1-4. Appl. Microbiol. Biotechnol. 2008, 78, 483-493. [CrossRef] [PubMed]

121. Mockaitis, G.; Braunt, G.; Foresti, E.; Zaiat, M.; Guiot, S.R. Microbial Communities Performing Hydrogen Solventogenic Metabolism of Volatile Fatty Acids. bioRxiv 2021. [CrossRef]

122. Yang, Y.; Nie, X.; Jiang, Y.; Yang, C.; Gu, Y.; Jiang, W. Metabolic regulation in solventogenic clostridia: Regulators, mechanisms and engineering. Biotechnol. Adv. 2018, 36, 905-914. [CrossRef] [PubMed]

123. Jia, X.; Xi, B.D.; Li, M.L.; Yang, Y.; Wang, Y. Meraptoreomic analysis of the functional insights into microbial communities of combined hydrogen and methane production by anaerobic fermentation from reed straw. PLoS ONE 2017, 12, e0183158. [CrossRef]

124. Ziganshin, A.M.; Ziganshina, E.E.; Kleinstruber, S.; Nikolausz, M. Comparative Analysis of Methanogenic Communities in Different Laboratory-Scale Anaerobic Digesters. Archaea 2016, 2016, 1-12. [CrossRef] [PubMed]

125. Hanreich, A.; Heyer, R.; Benndorf, D.; Rapp, E.; Pioch, M.; Reichl, U.; Klocke, M. Metaproteome analysis to determine the metabolically active part of a thermophilic microbial community producing biogas from agricultural biomass. Can. J. Microbiol. 2012, 58, 917-922. [CrossRef]

126. Zhang, J.; Liang, Y.; Yau, P.M.; Pandey, R.; Harpalani, S. A metaproteomic approach for identifying proteins in anaerobic bioreactors converting coal to methane. Int. J. Coal Geol. 2015, 146, 91-103. [CrossRef]

127. Heyer, R.; Benndorf, D.; Kohrs, F.; De Vrieze, J.; Boon, N.; Hoffmann, M.; Rapp, E.; Schlüter, A.; Sczyrba, A.; Reichl, U. Proteotyping of biogas plant microbiomes separates biogas plants according to process temperature and reactor type. Biotechnol. Biofuels 2016, 9, 1-16. [CrossRef] [PubMed]

128. Morris, R.; Schauer-Gimenez, A.; Bahattad, U.; Kearney, C.; Struble, C.A.; Zitomer, D.; Maki, J.S. Methyl coenzyme M reductase (mcrA) gene abundance correlates with activity measurements of methanogenic $\mathrm{H} 2 / \mathrm{CO} 2$-enriched anerobic biomass. Appl. Microbiol. 2013, 7, 77-84. [CrossRef]

129. Aguinaga Casañas, M.A.; Rangkasenee, N.; Krattenmacher, N.; Thaller, G.; Metges, C.C.; Kuhla, B. Methyl-coenzyme M reductase A as an indicator to estimate methane production from dairy cows. J. Dairy Sci. 2015, 98, 4074-4083. [CrossRef] [PubMed]

130. Munir, R.I.; Spicer, V.; Krokhin, O.V.; Shamshurin, D.; Zhang, X.; Taillefer, M.; Blunt, W.; Cicek, N.; Sparling, R.; Levin, D.B. Transcriptomic and proteomic analyses of core metabolism in Clostridium termitidis CT1112 during growth on $\alpha$-cellulose, xylan, cellobiose and xylose. BMC Microbiol. 2016, 16, 1-21. [CrossRef] [PubMed]

131. Kruse, S.; Goris, T.; Westermann, M.; Adrian, L.; Diekert, G. Hydrogen production by Sulfurospirillum species enables syntrophic interactions of Epsilonproteobacteria. Nat. Commun. 2018, 9, 1-13. [CrossRef]

132. Yan, Q.; Li, Y.; Huang, B.; Wang, A.; Zou, H.; Miao, H.; Li, R. Proteomic profiling of the acid tolerance response (ATR) during the enhanced biomethanation process from Taihu Blue Algae with butyrate stress on anaerobic sludge. J. Hazard. Mater. 2012, 235, 286-290. [CrossRef] [PubMed]

133. Roy, S.; Banerjee, D.; Dutta, M.; Das, D. Metabolically redirected biohydrogen pathway integrated with biomethanation for improved gaseous energy recovery. Fuel 2015, 158, 471-478. [CrossRef]

134. Dev, S.; Saha, S.; Kurade, M.B.; Salama, E.-S.; El-Dalatony, M.M.; Ha, G.S.; Woong, S.; Jeon, B.H. Perspective on anaerobic digestion for biomethanation in cold environments. Renew. Sustain. Energy Rev. 2019, 103, 85-95. [CrossRef] 
135. De Vrieze, J.; Hennebel, T.; Boon, N.; Verstraete, W. Methanosarcina: The rediscovered methanogen for heavy duty biomethanation. Bioresour. Technol. 2012, 112, 1-9. [CrossRef] [PubMed]

136. Pore, S.D.; Engineer, A.; Dagar, S.S.; Dhakephalkar, P.K. Meta-omics based analyses of microbiome involved in biomethanation of rice straw in a thermophilic anaerobic bioreactor under optimized conditions. Bioresour. Technol. 2019, 279, 25-33. [CrossRef] [PubMed]

137. Castellana, N.; Payne, S.; Shen, Z.; Stanke, M.; Bafna, V.; Briggs, S. Discovery and revision of Arabidopsis genes by proteogenomics. Proc. Natl. Acad. Sci. USA 2008, 105, 21034-21038. [CrossRef] [PubMed]

138. Mitsui, T.; Shiraya, T.; Kaneko, K.; Wada, K. Proteomics of rice grain under high temperature stress. Front. Plant Sci. 2013, 4, 1-5. [CrossRef] [PubMed]

139. Qiao, J.; Wang, J.; Chen, L.; Tian, X.; Huang, S.; Ren, X.; Zhang, W. Quantitative iTRAQ LC-MS/MS Proteomics Reveals Metabolic Responses to Biofuel Ethanol in Cyanobacterial Synechocystis sp. PCC 6803. J. Proteome Res. 2012, 11, 5286-5300. [CrossRef] [PubMed]

140. Tolonen, A.; Haas, W.; Chilaka, A.; Aach, J.; Gygi, S.; Church, G. Proteome-wide systems analysis of a cellulosic biofuel-producing microbe. Mol. Syst. Biol. 2011, 7, 461. [CrossRef] [PubMed]

141. Taillefer, M.; Arntzen, M.; Henrissat, B.; Pope, P.; Larsbrink, J. Proteomic Dissection of the Cellulolytic Machineries Used by Soil-Dwelling Bacteroidetes. Msystems 2018, 3, e00240-18. [CrossRef] [PubMed] 